\title{
ANALYTIC THEORY OF LINEAR DIFFERENTIAL EQUATIONS.
}

\author{
BY
}

\author{
W. J. TRJITZINSKY
}

of Evanston, III., U. S. A.

Index.

$I^{\circ}$. Introduction.

I. Preliminary Facts.

2. The $Q, Q_{x}$ and $C_{x}$ Curves and Regions $R$.

3. A Lemma concerning Regions $R_{i}$.

4. Formal Integration.

5. Analytic Integration.

6. Iterations.

7. The Fundamental Existence Theorem.

8. Extension of the Regions of Validity of the Asymptotic Relations.

9. Converse Problems.

$1^{\circ}$. Introduction. Our present object is to develop, on the basis of the formal solutions and without any restrictions on the roots of the corresponding characteristic equation, the analytic theory of a linear differential equation of order $n$

$$
\begin{gathered}
L_{n}(y) \equiv a_{0}(x) y^{(n)}(x)+a_{1}(x) y^{(n-1)}(x)+\cdots+a_{n-1}(x) y^{(1)}(x)+a_{n}(x) y(x)=0 \\
{\left[a_{0}(x) \neq 0 ; \quad a_{n}(x) \neq \mathrm{o}\right]}
\end{gathered}
$$

from the point of view of the asymptotic nature of the solutions. Such a study will be given for the neighborhood of a singular point (regular or irregular). This point will be taken at infinity. The coefficients in (A) will be supposed to be analytic for $|x| \geqq \varrho(|x|) \neq \infty)$, being representable by convergent series of the form

(1) $\quad \alpha(x)=a_{M} x^{\frac{M}{p}}+a_{M-1} x^{\frac{M-1}{p}}+\cdots+a_{1} x^{\frac{1}{p}}+a_{0}+a_{-1} x^{-\frac{1}{p}}+a_{-2} x^{-\frac{2}{p}}+\cdots$, 
where $p(\geqq \mathrm{I})$ is an integer. More generally, the coefficients in (A) may be allowed to be merely asymptotic in certain regions to series of the form(I). In this case these series may be divergent, and the results of the paper would apply, of course, to correspondingly restricted neighborhoods of infinity. It will be assumed that not all the coefficients of the series (I), which correspond to $a_{0}(x)$ and $a_{n}(x)$, are zero.

The development of a theory of an equation (A) is essentially equivalent to that of a system of order $n$,

$$
\begin{gathered}
Y^{(1)}(x)=Y(x) A(x), \quad Y(x)=\left(y_{i, j}(x)\right), \quad A(x)=\left(a_{i, j}(x)\right) \\
(i, j=\mathrm{I}, 2, \ldots n) .
\end{gathered}
$$

Here matrix notation is used with $a_{i, j}(x)$, for instance, denoting the element of the matrix $A(x)\left[=\left(a_{i, j}(x)\right)\right]$ in the $i$-th row and $j$-th column. The determinant $\left|\left(a_{i, j}(x)\right)\right| \neq 0$. The elements of a row in $Y(x)$ will denote a solution. As in the case of the coefficients in (A), the coefficients in (B) are either representable by convergent series of the form (I) or are asymtotic (in certain regions) to such, possibly divergent, series. In the case when $\left|\left(a_{i, j}\right)\right\rangle \mid$ is merely asymptotic to a series (I) it will be assumed that not all the coefficients in the series are zero.

An equation (A) or a system (B), of the above kind, will be said to be singular. Whenever necessary, it will be said that an equation or system is singular in a region $R$. It is evident that the class of singular equations includes every equation which has at infinity a singular point of (any) rank $K$.

Of the number of earlier treatments, of the type undertaken here, we shall mention the two papers most relevant to the present work. There is a paper by Poincaré ${ }^{1}$ in which an equation of rank one is treated. In a later paper, by G. D. Birkhoff ${ }^{2}$, the case of equations of any finite rank is considered. H. Poincaré employs Laplace integrals, and G. D. Birkhoff's work is based on certain generalizations of these integrals. The methods of these two writers, while applicable to the case of unequal roots of the characteristic equation, lead to indefinite algebraic complications when the treatment of the truly general case of unrestricted roots and any rank is attempted. Subsequent to the mentioned works of these two writers no additionally significant advances have been made in so far as the asymptotic nature of the solutions is concerned in the general

${ }^{1}$ H. Poincaré, American Journal of Mathematics, vol. 7 (I 885), pp. 203-258.

2 G. D. Brikhoff, Trans. Am. Math. Soc., vol. Io (1909), pp. 436-470. Cf. also J. Horn, Math. Zeit., vol. 2I (1924), pp. 85-95; here many references are given. 
case now under consideration. Several other well known recent works are important in certain directions different from those pursued in the present paper.

In dealing with the general case under consideration the present author found it necessary to develop and apply an entirely different method of attack. The key to this method is developed in section 6 concerning Iterations. Iterations here are of significance analogous to that of (being inspired by) the important method of iterations originally developed by G. D. Birkhoff in the field of difference equations.

The fields of differential, difference and $q$-difference equations are to a considerable degree analogous. In all three the case of restricted roots of the corresponding characteristic equation had been treated first. The unrestricted case of difference equations (from the point of view of asymptotic properties) has been treated in a joint paper by the present author and G. D. Birkhoff. ${ }^{1}$ The general case of $q$-difference equations has been treated by the present author. ${ }^{2}$ In this sense the present paper is to fill the remaining gap.

I. Preliminary Facts. A singular equation (A) will remain singular after division by $a_{0}(x)$. Accordingly, without any loss of generality we let $a_{0}(x)=\mathrm{I}$. Closely associated with the equation (A) is the system (which will be singular)

$$
\begin{aligned}
& Y^{(1)}(x)=Y(x) D(x), \quad Y(x)=\left(y_{i, j}(x)\right),
\end{aligned}
$$

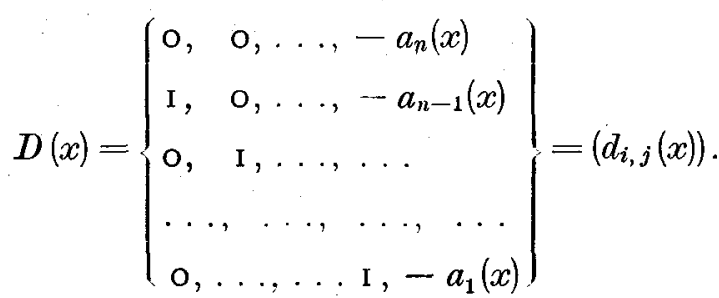

Whenever $Y(x)=\left(y_{i, j}(x)\right)$ is a matrix solution of $(\mathrm{D})$ it will necessarily follow that

$$
y_{i, j}(x)=y_{i, 1}^{(j-1)}(x) \quad(i, j=\mathrm{I}, 2, \ldots n)
$$

Moreover, the elements of the first column will constitute a full set of solutions of the equation (A). Conversely, if

${ }^{1}$ G. D. Birkhoff and W. J. Trjitzinsky, Analytic Theory of Singular Difference Equations, Acta mathematica, vol. 6o (I933), pp. I-89.

${ }^{2}$ W. J. Trjitzinsky, Analytic Theory of Linear q-difference Equations, Acta mathematica, vol. 6I (1933), pp. I-38.

22-33617. Acta mathematica. 62. Imprimé le 6 novembre 1933. 


$$
y_{i}(x)=y_{i, 1}(x) \quad(i=\mathrm{I}, 2, \ldots n)
$$

form a complete set of solutions of (A),

$$
Y(x)=\left(y_{i, j}(x)\right)=\left(y_{i, 1}^{(j-1)}(x)\right)
$$

will constitute a matrix solution of (D).

The non-homogeneous equation

$$
L_{n}(y)=z(x)
$$

will be satisfied by $y(x)$,

$$
y(x)=\sum_{x=1}^{n} y_{\lambda, 1}(x) \int z(x) \bar{y}_{n, \lambda}(x) d x
$$

where the elements $\bar{y}_{i, j}(x)$ are those of the inverse of the matrix $\left(y_{i, 1}^{(j-1)}(x)\right)$; that is,

$$
\left(\bar{y}_{i, j}(x)\right)=\left(y_{i, 1}^{(j-1)}(x)\right)^{-1} .
$$

This holds, of course, only inasmuch as the integrations in (4) remain valid.

A singular equation (A) will always possess a full set of formal series solutions of the form

$$
\begin{gathered}
s_{i}(x)=e^{Q_{i}(x)} x^{r_{i}} \sigma_{i}(x), \quad Q_{i}(x)=\sum_{v=0}^{r=l_{i}-1} q_{v}^{i} x^{\frac{l_{i}-v}{k_{i}}} \\
\sigma_{i}(x)=\sigma_{i}^{0}(x)+\sigma_{i}^{1}(x) \log x+\cdots+\sigma_{i}^{m_{i}}(x) \log { }^{m_{i}}(x),
\end{gathered}
$$

$$
\begin{gathered}
\sigma_{i}^{M I}(x)=\sigma_{i}^{M, 0}+\sigma_{i}^{H, 1} x^{-\frac{1}{k_{i}}}+\sigma_{i}^{M, 2} x^{-\frac{2}{k_{i}}}+\cdots \\
\left(M=0, \mathrm{I}, \ldots m_{i} ; \quad i=\mathrm{I}, 2, \ldots n\right)
\end{gathered}
$$

where $l_{i}, m_{i}, k_{i}$ are integers $\left(m_{i} \geqq 0 ; k_{i}=r_{i} p ;\right.$ integer $\left.r_{i} \geqq 1\right){ }^{1}$

Whenever any of the coefficients in (A) are merely asymptotic to series of the form $\left(\mathrm{I} ; \S \mathrm{I}^{\circ}\right)$ the formal solutions are those satisfying the modified formal equation $\left(A^{\prime}\right)$, which is obtained by replacing the coefficients in (A) by the series $\left(\mathrm{I} ; \S \mathrm{I}^{\circ}\right)$, to which these coefficients are asymptotic. The existence of a full set (that is, a linearly independent set) of $n$ formal series solutions of the type (5)

\footnotetext{
'Except with $x$ and $\log x$, superscripts here do not denote powers.
} 
is a consequence of the work of Fabry. ${ }^{\prime}$ These series, in general, do not converge. The formal series can be arranged in groups so that the exponential factors

$$
e^{Q(x)} x^{r}
$$

of the series belonging to a particular group are the same. ${ }^{2}$ On the other hand, the elements of such a group can and will be so ordered,

$$
e^{Q(x)} \dot{x}^{r} \sigma_{p+1}(x), e^{Q(x)} x^{r} \sigma_{p+2}(x), \ldots, e^{Q(x)} x^{r} \sigma_{p+q}(x)
$$

that $m_{p+1}=\mathrm{O}, m_{p+2}=\mathrm{I}, \ldots m_{p+q}=q-\mathrm{I}$. Such groups will be called logarithmic.

A series $\sigma(x)$ of the form (5 a), (5 b) will be termed a o-series.

Let $\mathrm{I} \leqq \Gamma<n$. If the first $\Gamma$ formal series (5) contain only complete logarithmic groups, the determinant of order $r$

$$
\left|\left(s_{i}^{(j-1)}(x)\right)\right| \quad\left(i, j={ }_{1}, 2, \ldots \Gamma\right)
$$

when formally computed as a series of the type (5), will contain no logarithms.

In the case of a system (B) we have a formal matrix solution

$$
S(x)=\left(s_{i, j}(x)\right)=\left(e^{Q_{i}(x)} x^{r_{i, j}} \sigma_{i, j}(x)\right)
$$

where the $\sigma_{i, j}(x) \quad(i, j=\mathrm{I}, \ldots n)$ are $\sigma$-series. For a fixed $i$ the numbers $r_{i, j}(j=1,2, \ldots n)$ may differ only by rational numbers. Facts analogous to those stated concerning the series (5) will hold for the elements of $S(x)$. Such a matrix can always be found so that, formally, the determinant $|S(x)|$ does not vanish.

2. The $Q, Q_{x}$ and $C_{x}$ Curves and Regions $R$. In the sequel it will be essential to consider branches, extending to infinity, along which

$$
\Re Q_{i, j}(z)=\mathrm{o} \quad\left[Q_{i, j}(z)=Q_{i}(z)-Q_{j}(z)\right]
$$

for some $i$ and $j(i \neq j)$. Such a branch will be termed a $Q$ curve. ${ }^{3}$

${ }^{1}$ E. Fabry, Sur les intégrales des équations différentielles linéaires à coefficients rationnels, Thèse, 1885, Paris.

${ }^{2}$ Except, possibly, the values of $r$, associated with the same group, may differ by rational fractions.

${ }^{3}$ If $c=a+V=1 b, \Re e=a$. 
We are interested in the properties of a $Q$ curve in the neighborhood of infinity. On writing

$$
\begin{gathered}
Q(z)=\sum_{\nu=0}^{r=l-1} q_{v} z^{l-\vec{k}} \\
{\left[q_{v}=\left|q_{v}\right| e^{v-1} q_{\nu} ; \quad z=v e^{v-1 \theta} ; \quad q_{0} \neq 0 ; \quad l \geqq \mathrm{I}\right],}
\end{gathered}
$$

it follows that

$$
\Re Q(z)=\sum_{v=0}^{l-1}\left|q_{\nu}\right| r^{\frac{l-v}{k}} \cos \left[\bar{q}_{v}+\left(\frac{l-v}{k}\right) \theta\right]
$$

Along the particular curve $Q$ under consideration

(4) $r^{-\frac{l}{k}} \Re Q(z)=\left|q_{0}\right| \cos \left(\bar{q}_{0}+\frac{l}{k} \theta\right)+\sum_{v=1}^{l-1}\left|q_{v}\right| r^{-\frac{r}{k}} \cos \left[\bar{q}_{v}+\left(\frac{l-\nu}{k}\right) \theta\right]=0$.

Thus, along $Q$,

$$
\left|q_{0}\right| \cos \left(\vec{q}_{0}+\frac{l}{k} \theta\right)=-\sum_{v=1}^{l-1}\left|q_{v}\right| r^{-\frac{r}{k}} \cos \left[\bar{q}_{v}+\left(\frac{l-\nu}{k}\right) \theta\right] .
$$

In $(4$ a) let $r \rightarrow \infty$. Hence, on writing the equation of $Q$ in the form

$$
\theta=\theta(r) \text {, }
$$

it follows that

$$
\lim \left|q_{0}\right| \cos \left(\bar{q}_{0}+\frac{l}{k} \theta(r)\right)=0
$$

Consequently every $Q$ curve is of the form

$$
\theta=r_{0}+p_{1}(r)
$$

where $r_{0}$ is a value such that

$$
\cos \left(\bar{q}_{0}+\frac{l}{k} r_{0}\right)=0
$$

and $\lim p_{1}(r)=0$. In other words, erery $Q$ curve has a limiting direction $\theta=r_{0}$ at infinity which is given by $(5 \mathrm{~b})$. The various $Q$ curves, satisfying the equation $\Re Q(z)=0$, have their limiting directions all distinct from each other. 
If

(5 c) $\quad \cos \left(\bar{q}_{0}+\frac{l}{k} r_{0}\right)=\cos \left(\bar{q}_{1}+\frac{l-\mathrm{I}}{k} r_{0}\right)=\cdots=\cos \left(\bar{q}_{l-1}+\frac{1}{k} r_{0}\right)=0^{1}$

it is clear that the $Q$ curve will consist of the ray $\theta=r_{0}$.

Suppose now that

(6) $\quad \cos \left(\bar{q}_{0}+\frac{l}{k} r_{0}\right)=\cos \left(\bar{q}_{1}+\frac{l-\mathrm{I}}{k} r_{0}\right)=\cdots=\cos \left(\bar{q}_{t-1}+\frac{l-t+\mathrm{I}}{k} r_{0}\right)=0$

$$
(0 \leqq t-\mathrm{I}<l-\mathrm{I})
$$

while

(6 a)

$$
\cos \left(\bar{q}_{t}+\frac{l-t}{k} r_{0}\right) \neq 0, \quad q_{t} \neq 0
$$

On substituting (5 a) we have, corresponding to every power $x^{\frac{j}{k}}(l \geqq j \geqq l-t+1)$ which actually enters in $Q(z)$,

(7) $\cos \left[\bar{q}_{j}+\frac{j}{k} r_{0}+\frac{j}{k} p_{1}(r)\right]=-\sin \left(\bar{q}_{j}+\frac{j}{k} r_{0}\right) \sin \frac{j}{k} p_{1}(r)=g_{j} \sin \frac{j}{k} p_{1}(v)$.

In $(7) g_{j}=-1$, whenever

$$
\bar{q}_{j}+\frac{j}{k} r_{0}=\frac{\pi}{2}+2 \lambda \pi \quad[\lambda, \text { an integer }]
$$

In the contrary case $g_{j}=\mathrm{I}$.

Thus, in the case of $(6)$ and $(6$ a) substitution of $(5$ a) in $(4)$ will give, in virtue of $(7)$ and for $z$ on $Q$,

(8) $\quad \frac{\mathrm{I}}{p_{1}(v)} r^{-\frac{l}{k}} \Re Q(z)=\left|q_{0}\right| g_{l} \frac{\sin \left(\frac{l}{k} p_{1}(r)\right)}{p_{1}(v)}+\sum_{j=l-1}^{j=l-t+1}\left|q_{l-j}\right| g_{j} r^{-\left(\frac{l-j}{k}\right)} \frac{\sin \left(\frac{j}{k} p_{1}(r)\right)}{p_{1}(v)}+$

$$
\begin{aligned}
& +\frac{r^{-\frac{t}{k}}}{p_{1}(r)}\left\{\left|q_{t}\right| \cos \left[\bar{q}_{t}+\frac{l-t}{k} r_{0}+\frac{l-t}{k} p_{1}(r)\right]+\sum_{x=1}^{x=l-1-t}\left|q_{t+x}\right| r^{-\frac{x}{k}}\right. \\
& \left.\cdot \cos \left[\bar{q}_{t+x}+\frac{l-t-x}{k} r_{\nu}+\frac{l-t-x}{x} p_{1}(r)\right]\right\}=0 .
\end{aligned}
$$

${ }^{1}$ The members corresponding to terms in $Q(z)$ not actually present are to be omitted. 
In particular, (8) will hold in the limit as $r \rightarrow \infty$. Accordingly,

$$
\left|q_{0}\right| g_{l} \lim _{r} \frac{\sin \left(\frac{l}{k} p_{1}(r)\right)}{p_{1}(r)}+\left|q_{t}\right| \cos \left(\bar{q}_{t}+\frac{l-t}{k} r_{0}\right) \lim _{r} \frac{r^{-\frac{t}{k}}}{p_{1}(r)}=0
$$

so that

$$
\lim _{r} \frac{r^{-\frac{t}{k}}}{p_{1}(r)}=\frac{-\left|q_{\mathrm{n}}\right| g_{l} l}{k\left|q_{t}\right| \cos \left(\bar{q}_{t}+\frac{l-t}{k} r_{0}\right)}=\frac{1}{r_{1}}
$$

From $(8$ a) and in view of $(6$ a) it follows that the curve $Q$ is of the form

$$
\theta=\theta(r)=r_{0}+r_{1} r^{-\frac{t}{k}}(\mathrm{I}+\varepsilon(r)) \quad\left(\mathrm{I} \leqq t \leqq l ; r_{1} \neq \mathrm{o}\right),
$$

where $\lim _{r} \varepsilon(r)=\mathrm{O}$ (in the case when it does not consist of the ray $\theta=r_{0}$; that is, provided (6), (6 a) hold).

Let the position of any point $P$ on the curve $Q$ be defined by a pair of numbers, $(u, v)$, where $v$ is the distance from $P$ to the ray $\theta=r_{0}$ and $u$ is the distance from the origin to the foot of the perpendicular from $P$ to the ray. The convention will be made that $v>0$ when $p_{1}(r)>0$ and that $v<0$ whenever $p_{1}(v)<0$. It will follow then that

$$
r=u \sqrt{\mathrm{I}+\left(\frac{v}{u}\right)^{2}}=u\left(\mathrm{I}+\varepsilon_{1}(r)\right) \quad\left(\lim _{r} \varepsilon_{1}(r)=\mathrm{o}\right),
$$

since in view of the relation

$$
\frac{v}{u}=\operatorname{tg} p_{1}(r)=r_{1} r^{-\frac{t}{k}}\left(\mathrm{I}+\varepsilon_{2}(r)\right) \quad\left(\lim \varepsilon_{2}(r)=0\right)
$$

we have

$$
\lim _{r}\left(\frac{v}{u}\right)=0
$$

From (9)

$$
r^{-{ }^{t}}=u^{-\frac{t}{k}}\left(\mathrm{I}+\varepsilon_{3}(r)\right) \quad\left(\lim _{r} \varepsilon_{3}(r)=0\right)
$$

Thus, on taking account of $(9$ a) and of the fact that $u$ and $r$ approach infinity simultaneously, every curve $Q$ is seen to be of the form 
(10)

$$
\begin{gathered}
v=r_{1} u^{\frac{k-t}{k}}(\mathrm{I}+\xi(u)) \\
{\left[\mathrm{I}>\frac{k-t}{k} \geq \frac{k-l}{k} ; \quad \lim _{u} \xi(u)=\mathrm{o}\right.}
\end{gathered}
$$

(with the rectangular coordinates $(u, v)$ taken with respect to the ray $\theta=r_{0}$, as the $U$ axis).

Thus a $Q$ curve is either the ray $\theta=r_{0}$ (whenever $(5 c)$ holds) or it is the ray $v=r_{1} \neq 0$ (whenever $k=t$ and $\xi(u)$ in (Io) is zero). If the curve does not consist of any of these rays, it will be either asymptotic to the ray $\theta=r_{0}$ (whenever $0>k-t$ ) or it will be asymptotic to the ray $v=r_{1}$ (whenever $o=k-t$ ) or it will possess no asymptote. The latter will be the case whenever $k-t>0$; the curve will then recede indefinitely from the ray $\theta=r_{0}$.

$Q$ curves form a special ease of $Q_{x}$ curves. $A Q_{x}$ curve will be defined as a z-branch extending to infinity along which

$$
\Re Q(z)=q[=\Re Q(x)] .
$$

Such a curve extends from $z=x$. The equation (I I), with $q=0$, will define $Q$ curves.

We shall write

$$
r^{-\frac{1}{k}}=\Im
$$

The equation (II) can be expressed in the form

(I I b) $\quad r^{-\frac{l}{k}}[\Re Q(z)-q] \equiv \sum_{v=0}^{l-1}\left|q_{v}\right| \cos \left[\bar{q}_{v}+\frac{l-v}{k} \theta\right] \Im^{v}-q \mathfrak{I}^{l} \equiv f_{1}(\theta, \mathfrak{\Im})=0$.

On noting that

$$
f_{1}(\theta, o)=\left|q_{0}\right| \cos \left(\bar{q}_{0}+\frac{l}{k} \theta\right)
$$

it is observed that the equation $f_{1}(\theta, 0)=0$ has only real, simple roots. These values are identical with the limiting directions, obtained before, of the various $Q$ curves, satisfying the equation $\Re Q(z)=0$. Let $\theta=r_{0}$. be one of these values. The third member in ( I I $b$ ) is entire in $\eta=\theta-r_{0}$ and $\mathfrak{J}$. Thus,

$$
f_{1}(\theta, \Im) \equiv f(\eta, \Im)=\sum_{k=0}^{\infty} \sum_{\nu=0}^{l} f_{n, \nu} \eta^{n} \Im^{v} \quad\left(f_{0,0}=0\right)
$$


Since

$$
f(\eta, o)=\left|q_{0}\right| \cos \left(\bar{q}_{0}+\frac{l}{k} \eta+\frac{l}{k} r_{0}\right)
$$

it follows that

(I I e)

$$
f_{1,0}=\frac{l h\left|q_{0}\right|}{k} \neq \mathrm{o} \quad\left[h=-\sin \left(\bar{q}_{0}+\frac{l}{k} r_{0}\right)= \pm \mathrm{I}\right]
$$

On the other hand,

$$
f(o, \mathfrak{J})=f_{1}\left(r_{0}, \mathfrak{I}\right)=\sum_{r=1}^{l-1}\left|q_{v}\right| \cos \left[\vec{q}_{v}+\left(\frac{l-v}{k}\right) v_{0}\right] \mathfrak{J}^{v}-q \mathfrak{\Im}^{l}=\sum_{v=1}^{l} f_{0, v} \mathfrak{\Im}^{r}
$$

Hence we may write

$$
f_{0, \sigma}=\left|q_{\sigma}\right| \cos \left(\bar{q}_{\sigma}+\frac{l-\sigma}{k} r_{0}\right) \quad(\sigma=\mathrm{I}, \ldots l-\mathrm{I})
$$

$$
f_{0, l}=-q, \quad f_{0, l+x}=0 \quad(x=1,2, \ldots)
$$

By the theory of implicit functions to the pair of values $\eta=0$ (that is, $\left.\theta=r_{0}\right), \mathfrak{J}=0$ there corresponds a solution satisfying (l $\left.\begin{array}{ll}\text { I } & b\end{array}\right)$,

$$
\theta-r_{0}=\eta=\eta_{1} \mathfrak{\Im}+\eta_{2} \mathfrak{\Im}^{2}+\cdots
$$

The series in the last member of (I2) converges in the complex $\Im$-plane for $|\mathfrak{I}| \leqq \mathfrak{I}_{0}\left(\mathfrak{S}_{0}>0\right)$. Since the $f_{n, \nu}$ are real it follows easily that the $\eta_{i}(i=1,2, \ldots)$ are also real. To examine the $\eta_{i}$ in greater detail (12) will be substituted in ( 1 i b). Thus,

$$
\sum_{n=0}^{\infty} \sum_{v=0}^{l} f_{n, v}\left(\eta_{1} \mathfrak{\Im}+\eta_{2} \mathfrak{S}^{2}+\cdots\right)^{n} \mathfrak{J}^{v}=0
$$

In view of satisfied conditions of convergence, rearrangement of terms is possible. We obtain

so that

$$
\sum_{m=1}^{\infty} f_{m} \mathfrak{\Im}^{m} \equiv 0
$$

$$
f_{1}=f_{1,0} \eta_{1}+f_{0,1}=0
$$

$$
\begin{gathered}
f_{m}=f_{1,0} \eta_{m}+h_{m}\left(\eta_{1}, \eta_{2}, \ldots \eta_{m-1}\right)+f_{0, m}=0 \\
(m=2,3, \ldots) .
\end{gathered}
$$


Here the functions $h_{m}\left(\eta_{1}, \eta_{2}, \ldots \eta_{m-1}\right)$ are real for $\eta_{1}, \eta_{2}, \ldots \eta_{m-1}$ real, they do not involve the constants $f_{0, i}$ and hence they are independent of $q$; moreover, $h_{m}(\mathrm{o}, \mathrm{o}, \ldots \mathrm{o})=\mathrm{o}$.

Consider the case of (6), (6 a). Successive application of (13) and (I 3 a) will give

$$
\eta_{1}=\eta_{2}=\cdots=\eta_{t-1}=\mathrm{o}
$$

From ( 3 a; $m=t$ ) it will follow that

$$
\eta_{t}=\frac{-f_{0, t}}{f_{1,0}} \neq \mathrm{o}
$$

In view of (I I e), ( I I f) and ( 6 a), this agrees with the corresponding result obtained before $\left(r_{1}=\eta_{t}\right)$. In general, successive application of (I3 a), for $m=t, \ldots l-\mathrm{I}$, will determine uniquely the constants $\eta_{t}, \eta_{t+1}, \ldots \eta_{l-1}$. These constants will be independent of $q$ since the equations ( I 3$),($ I $3 a ; m=2,3, \ldots l-$ I) do not contain $q$. On the other hand, $\eta_{l}, \eta_{l+1}, \ldots$ will involve $q$. In fact,

so that

$$
f_{1,0} \eta_{l}+h_{l}\left(\eta_{1}, \ldots \eta_{l-1}\right)-q=\mathrm{o}
$$

$$
\eta_{l}=w q+\eta_{l}^{\prime \prime} \quad\left(w=\frac{k}{l h\left|q_{0}\right|} \neq 0\right)
$$

where $\eta_{i}$ is independent of $q$.

Thus, in the case of (6), (6 a) the $Q_{x}$ curve, whose limiting direction at infinity is $r_{0}$ and which satisfies the equation ( $\mathrm{I} \mathrm{I}$ ), is given by the equation

$$
\text { (I 4) } \begin{aligned}
\theta=\theta_{x}=r_{0}+\eta_{t} r^{-\frac{t}{k}}+\eta_{t+1} r^{-\left(\frac{t+1}{k}\right)}+\cdots+\eta_{l-1} r^{-\left(\frac{l-1}{k}\right)}+\left(w q+\eta_{l}^{0}\right) r^{-l} & +\cdots \\
& +\eta_{l+1} r^{-\left(\frac{l+1}{k}\right)}+\cdots
\end{aligned}
$$

$\left[\eta_{t}, \eta_{t+1}, \ldots \eta_{l-1}, \eta_{i}^{0}\right.$ independent of $\left.x ; z=r e^{-1 \theta}\right]$, the series being convergent for $r \geqq r^{\prime}>0$. In the case of (5 c) in place of (14) we have

$$
\theta=\theta_{x}=r_{0}+\left(w q+\eta_{l}^{0}\right) r^{-\frac{l}{k}}+\eta_{l+1} r^{-\left(\frac{l+1}{k}\right)}+\cdots \quad\left(r \geqq r^{\prime}\right)
$$

It is observed, then, that $a Q_{x}$ curve has always the limiting direction of $a$ corresponding $Q$ curve. On letting $q=0$ in (14) a $Q$ curve, whose limiting direction 23-33617. Acta mathematica. 62. Imprimé le 7 novembre 1933. 
is $r_{0}$ and which does not consist of the ray $\theta=r_{0}$, is seen to be expressible in the form

(14 b) $\quad \theta=r_{0}+\eta_{t} r^{-\frac{t}{k}}+\eta_{t+1} r^{-\left(\frac{t+1}{k}\right)}+\cdots+\eta_{l-1} r^{-\left(\frac{l-1}{k}\right)}+\eta_{i}^{0} r^{-\frac{l}{k}}$

$$
\left(z=r e^{v-1 \theta}\right) \text {. }
$$

Let $z_{x}=r e^{\gamma-1} \theta_{x}$ be on the curve $Q_{x}$ given by (14) or (I4 a); let $z=r e^{\gamma-1} \theta$ be on the corresponding $Q$ curve. Then

$$
\text { (I4 c) } \quad \theta_{x}-\theta=w q r^{-\frac{l}{k}}+\eta_{l+1}^{\prime} r^{-\left(\frac{l+1}{k}\right)}+
$$

Consider a function $\Re Q(z)$ which vanishes along a $Q$ curve, whose equation is $\theta=\theta(r)$. $\Re Q(z)$ will be positive in some region $R$, extending to infinity and lying to one side of $Q$. With $r$ fixed $(r \geqq e)$

$$
\text { H } Q\left(r e^{1-1 \theta}\right)
$$

will increase monotonically as $\theta$ varies from $\theta=\theta(r)$ to some value $\theta=\theta^{*}(r)$ $\left[\theta(r) \gtrless \theta^{*}(r)\right.$, as the case may be]. The value $\theta^{*}(r)$ could be defined as the root $\theta$ satisfying the equation

$$
\frac{\partial}{\partial \theta} \Re Q\left(\cdot e^{y^{\prime-1} \theta}\right)=0
$$

and lying nearest to $\theta=\theta(r)$ (from the side under consideration). A $Q^{*}$ curve will be defined as a branch extending to intinity and satisfying ( 15 a). According to the preceding $\theta=\theta^{*}(r)$ defines a $Q^{*}$ curve. Now

$$
-r^{-\frac{l}{k}} \frac{\partial}{\partial \theta} \Re Q(z) \equiv \frac{l}{k}\left|q_{0}\right| \sin \left(\bar{q}_{0}+\frac{l}{k} \theta\right)+\sum_{v=1}^{l-1} \frac{l-v}{k}\left|q_{v}\right| r^{-\frac{v}{k}} \sin \left(\bar{q}_{v}+\frac{l-v}{k} \theta\right)=0
$$

so that the limiting directions of all possible $Q^{*}$ curves, satisfying ( 15 a), are given by the roots $r_{0}^{*}$ of the equation

$$
\sin \left(\bar{q}_{0}+\frac{l}{k} r\right)=0
$$

Thus, the limiting directions of the $Q^{*}$ curves, satisfying ( 15 a), are all distinct from those of the several $Q$ curves satisfying the equation $\Re Q(z)=0$. The 
region bounded by a $Q$ curve and by a certain one of the $Q^{*}$ curves will have the property that $\Re Q(z)$ increases monotonically, as $z$ describes any circular $\operatorname{arc}|z|=r(\geqq \varrho)$ from the $Q$ curve to the $Q^{*}$ curve.

Corresponding to those of the functions $\Re Q_{i, j}(z)$ [cf. (I)] which are not identically zero we have a finite set of $Q$ curves. It is conceivable that some of the $Q$ curves are 'multiple'; that is, that there exist two (or more) functions $\Re Q(z)$ vanishing along precisely the same curve. In view of the preceding it is clear that for $|z|=r \geqq \varrho$ ( $\varrho$ sufficiently great) the various distinct $Q$ curves have no points in common (for $r \neq \infty$ ). Accordingly, the complete vicinity of infinity

$$
\theta_{0} \leqq \theta=\arg x \leqq 2 \pi k+\theta_{0} \quad\left(|x|=r \geqq \varrho_{0}>0\right),
$$

is divided in a number of suecessively adjacent regions

$$
R_{1}, R_{2}, \ldots R_{N}
$$

separated by $Q$ curves and not containing any $Q$ curves in the interior. We let $B_{i, i+1}$ (or $B_{i+1, i}$, as well) denote the $Q$ curve (simple, or multiple) which constitutes the common boundary of $R_{i}$ and $R_{i+1}$. The region $R_{1}$ will have a $Q$ curve, $B_{1, N}$, in common with $R_{N}$. The importance of these regions is due to the fact that within any particular region $R_{i}$ a certain ordering of the values $\Re Q_{i}(z)$ is maintained. Consider a particular function $\Re Q(z)\left[=\Re Q_{i, j}(z)=\right.$ $\left.=\Re Q_{i}(z)-\Re Q_{j}(z) \neq \mathrm{o}\right]$. We let $R(Q)$ (or $R\left(Q_{i, j}\right)$ ) denote any one of the several regions extending to infinity ${ }^{1}$, throughout which $\mathfrak{R} Q(z)$ is non-negative.

Furthermore, any subregion of $R(Q)$ will be denoted by $\mathbb{R}(Q)$, provided that it is bounded on both sides by regular curves ${ }^{2}$ extending to infinity, possessing limiting directions at infinity ${ }^{3}$, such that along each of these boundaires

$$
|z|^{-\beta} e^{\Re Q(z)} \rightarrow \infty
$$

$$
\text { (as }|z| \rightarrow \infty \text {; for every positive } \beta \text { ); }
$$

\footnotetext{
1 Speaking of various regions extending to infinity, the shape of the boundary near the origin is immaterial. We may always consider this part of the boundary as consisting of a circular are $r=\varrho_{1}>0\left(\varrho_{1}\right.$ being sufficiently great $)$.

${ }^{2} \mathrm{~A}$ curve will be said to be regular if it is representable by an equation of the form

$$
\theta=c_{0}+c_{1} r^{-\frac{1}{k_{1}}}+c_{2} r^{-\frac{2}{k_{1}}}+\cdots \quad\left(k_{1}\right. \text { some integer). }
$$

3 These directions can be always taken coincident with those of the corresponding $Q$ curves.
} 
(I 7$)$ will hold, for every positive $\beta$, along every path lying in $R(Q)$ and possessing a limiting direction.

Let $R_{i}$ denote any particular one of the regions ( 16 a), whose boundaries have different limiting directions. Let

$$
Q_{i_{x}, j_{x}}(z) \equiv Q_{i_{x}}(z)-Q_{j_{x}}(z) \quad(\not \neq 0 ; \varkappa=1,2, \ldots \lambda)
$$

constitute the totality of differences $\left(Q_{i}(z)-Q_{j}(z)\right)$, which have a non-negative real part in $R_{i}$ and which are such that for some $\beta$

$$
|z|^{-i} e^{\Re Q_{i_{x}}, j_{x}(z)} \rightarrow 0
$$$$
(x=1,2, \ldots \lambda)
$$

along both boundaries of $R_{i}$. It may happen that no such functions (I 8) exist. Suppose there are some functions of this kind. We let, then, $R^{\prime}{ }_{i}$ denote a subregion of $R_{i}$ with one boundary in common with $R_{i}$ and another boundary, interior $R_{i}$, such that along it all the left members of ( 18 a) increase indefinitely for every $\beta$ $(>0)$. We let $R_{i}^{\prime \prime}$ denote an analogous subregion of $R_{i}$, with the other boundary in common with $R_{i}$. $R_{i}^{\prime}$ and $R_{i}^{\prime \prime}$ can be chosen so as to overlap. In particular, $R_{i}^{\prime}$ can be so chosen that the limiting directions of its boundaries will be correspondingly the same as those of $R_{i}$; the same can be said of $R_{i}^{\prime \prime}$.

3. A Lemma concerning Regions $R_{i}(\mathrm{I} 6 \mathrm{a} ; \S 2)$. The following Definition will be introduced.

Definition. A region $R$, bounded by two regular curves, will be said to be proper if a certain ordering, say

$$
\Re Q_{1}(z) \leqq \Re Q_{2}(z) \leq \cdots \leqq \Re Q_{n}(z)
$$

is maintained for $z$ in $R$ and if the following holds. Corresponding to any $x$, in $R$, there exists a regular curve $C_{x}$, situated in $R$ and extending from $x$ to infinity, such that for every $\Re Q(z)\left\{=\Re Q_{i}(z)-\Re Q_{j}(z)\right]$, which is positive interior $R$, we have the following satisfied:

$I^{\circ} . \Re Q(z)$ is monotone non-decreasing for $|z| \geqq z(x)$ (where $z(x)$ is some number $\geqq|x|)$, as $|z| \rightarrow \infty$ along $C_{x}$; or

$2^{\circ}$. $\Re Q(z)$ is monotone decreasing for $|z| \geqq z(x)$ (where $z(x)$ is some number $\geqq|x|$ ), as $|z| \rightarrow \infty$ along $C_{x}$, while $\Re Q(z)$ is bounded in $R$.

$3^{\circ}$.

$$
\int_{x}\left|z^{-b} d z\right| \leqq \frac{c}{|x|^{b-i}}
$$

( $c$ and $\lambda$ independent of $x$ and $b ; b$ sufficiently great). 
$4^{\circ}$. Let $x=x_{0}^{\prime}, x_{1}, x_{1}^{\prime}, x_{2}, x_{2}^{\prime}, \ldots$ be any set of consecutive points on $C_{x}$, with some of them possibly coincident. The following condition holds

$$
h(x)=\sum \Re Q\left(x_{n}\right)-\mathfrak{R} Q\left(x_{n}^{\prime}\right) \leqq h
$$

( $h$, independent of $x$ and of the choice of the points).

The summation in (2) is only over all those points $x_{n}, x_{n}^{\prime}$ which in absolute value do not exceed $z(x)$.

With this definition in view the following Lemma, essential for our purposes, will be proved.

Lemma 1. Every region $R$ consisting of a region $R_{i}$ (cf. ( 16 a); $\$ 2$ ) or of a subregion of $R_{i}$, bounded by two regular curves, is proper or it consists of a finite number of proper adjacent or overlapping proper regions.

Let the boundaries, extending to infinity, of $R$ be $B$ and $B^{\prime}$. Suppose that, if $r e^{\gamma-1} \alpha$ is on $B$ and $r e^{r-1} \alpha^{\prime}$ is on $B^{\prime}$, we have $\alpha>\alpha^{\prime}(r \neq \infty)$. For convenience $B$ and $B^{\prime}$ will be termed left and right boundaries of $R$. The points $x$ in $R$ will all be such that $|x| \geqq \varrho_{1}>0$ where $\varrho_{1}$, so start with, is sufficiently great.

Case A. The boundaries $B, B^{\prime}$ have the same limiting direction. Until stated otherwise, only those $Q$ curves will be considered in the discussion of this case which have the same limiting direction as $B$ and $B^{\prime}$. Let $Q$ denote any such curve to the left of or coincident with $B$ and let $Q^{\prime}$ denote any such curve to the right of or coincident with $B^{\prime}$. By $\$ 2$ it follows that, if $\mathfrak{R} Q(z)$ corresponds to a curve $Q$, we shall have $\Re Q(z)$ increasing monotonically as $z$ moves from $B$ to $B^{\prime}$ so that $|z|\left(\geqq \varrho_{1}\right)$ remains fixed. A corresponding fact holds for any function $\Re Q^{\prime}(z)$, corresponding to a curve $Q^{\prime}$. In order that $\Re Q(z)$ be bounded in $R$ it is necessary and sufficient that $\Re Q(z)$ be bounded along $B^{\prime}$; similarly, for boundedness in $R$ of a function $\Re Q^{\prime}(z)$ it is necessary and sufficient that $\Re Q(z)$ be bounded along $B$. The functions under consideration, the $\mathfrak{R} Q(z)$ and the $\mathfrak{R} Q^{\prime}(z)$, are accordingly separated into two groups - those bounded in $R$,

$$
\mathfrak{R} Q^{b}(z), \quad \Re Q^{\prime b}(z)
$$

and others, not bounded in $R$,

$$
\Re Q^{u}(z), \quad \Re Q^{\prime}(z)
$$


Corresponding to an interior point $x$ of $R$ there will exist a group of $Q_{x}$ curves,

$$
Q_{x}^{u}, \quad Q_{x}^{\prime u}
$$

(cf. $\$ 2$ ) associated with the functions (4), respectively. These curves will extend from $x$ to infinity. Suppose that for no $x$, interior $R$, is a curve $Q_{x}^{u}$ situated to the right of a curve $Q_{x}^{\prime}$, sufficiently far from the origin. Let $Q_{x}^{u}$ denote one of the curves $Q_{x}^{\prime \prime}$ which, sufficiently far from the origin, is on or to the right of all the curves $Q_{x}^{u}$. Similarly, let $Q_{x}^{\prime \prime}$ denote a curre $Q_{x}^{\prime} u$ which sufficiently far out is on or to the left of all the curves $Q_{x}^{\prime \prime}$. $\quad \bar{Q}_{x}^{u}$ and $\bar{Q}_{x}^{\prime} u$ are either coincident or $\bar{Q}_{x}^{u}$ is to the left of $\bar{Q}_{x}^{u}$, sufficiently far from the origin. Let $x^{\prime}\left(\left|x^{\prime}\right|=|x|\right.$ be a point on $B^{\prime}$. At $x^{\prime} \Re \bar{Q}^{u}(z)$ is greater than at $x$; hence the curves $\left(_{x}^{u}\right.$ and $Q_{x^{\prime}}^{u}$ do not intersect. On the other hand, since $\mathfrak{R} Q^{u}(z)$ increases monotonically (and necessarily to infinity) along $B^{\prime}$ while for $z$ on $Q_{x^{\prime}}^{\prime \prime}$ we have $\Re \dddot{Q}^{u}(z)=$ $=\Re \bar{Q}^{u}\left(x^{\prime}\right)$, the curve $\bar{Q}_{x^{\prime}}^{u}$ recedes to the left from $B^{\prime}$. Therefore $\bar{Q}_{x}^{u}(x$ interior $R)$ has no points in common with $B^{\prime}$. Similarly, $Q_{x}^{\prime \prime}(x$ interior $R)$ will have no points in common with $B$. For a curve $C_{x}$, in $R$, extending to infinity and lying (sufficiently far out) between $Q_{x}^{u}$ and $\bar{Q}_{x}^{\prime}$, conditions $\mathrm{I}^{\circ}$ and $2^{\circ}$ of the Definition will be satisfied for the functions (4) as well as for the functions (3). Under the supposition of the italicized statement above such a curve (or curves) $C_{x}$ could be found for every $x$ interior $R$. There is no difficulty in seeing that this will remain true also for every $x$ on $B$ or $B^{\prime}$.

Assume that for some $x=x^{0}$, in $R$, a curve $Q_{x^{0}}^{u}$ (of the set (4)) is to the right of a curve $Q_{x^{0}}^{\prime \prime}$ (sufficiently far from the origin). We shall have one or more curves $Q_{x^{0}}^{u}$ and one or more curves $Q_{x^{0}}^{u}$ such that these $Q_{x^{0}}^{u}$ are all to the right of these curves $Q_{x^{0}}^{\prime \prime}$ (for $|z| \geqq \varrho\left(x_{0}\right) \geqq\left|x_{0}\right| ; \varrho\left(x_{0}\right)$ sufficiently great). Let $\tilde{Q}_{x^{\prime}}^{\prime u}$ be a curve, of the mentioned set of curves $Q_{x^{\prime}}^{\prime}$, which is on or to the right of these $\mathcal{Q}_{x^{0}}^{\prime \prime}$. Similarly, let $\tilde{Q}_{x^{0}}^{u}$ be a curve, of the mentioned set of curves situated on or to the left of all the curves of this set. It is clear that, for $|z| \geqq \varrho\left(x_{0}\right)$, $\tilde{Q}_{x^{\prime}}^{u}$ is to the left of $\tilde{Q}_{x^{0}}^{u}$. Let $x\left(|x|=\left|x^{0}\right|\right)$ be a point on $B$. The curves $B$, $\tilde{Q}_{x}^{\prime}{ }^{u}, \tilde{Q}_{x^{0}}{ }^{u}, \tilde{Q}_{x^{0}}^{u}$ will be in the order just stated, from the left to the right. This follows from the fact that along $B \mathfrak{R} \tilde{Q}^{\prime} u(z)$ increases and that $\mathfrak{R} \tilde{Q}^{\prime} u(z)$ is greater at $x$ than at $x^{0}$ (unless $\left.x=x^{0}\right)$. Thus, $B$ and $\tilde{Q}_{x^{0}}^{u}$ do not intersect for $|z| \geqq \varrho\left(x_{0}\right)$. For similar reasons $B^{\prime}$ and $\tilde{Q}_{x^{0}}^{\prime \prime}$ do not intersect $\left(|z| \geqq \varrho\left(x_{0}\right)\right)$. Accordingly, $R$ $\left(|z| \geqq \varrho\left(x_{0}\right)\right)$ is seen to consist of two overlapping regions; one, bounded by $B$ 
and $\tilde{Q}_{x^{0}}^{u}$; another, bounded by $\tilde{Q}_{x^{0}}^{\prime u}$ and $B^{\prime}$. Let $R_{1}$ stand for any particular one of these two regions. With respect to such a region $R_{1}$ the $\Re Q(z)$ and the $\Re Q^{\prime}(z)$ are separated into two groups:
$(3$,
$\Re Q_{1}^{b}(z)$,
$\Re Q_{1}^{\prime b}(z)$
(bounded in $R_{1}$ ),

and

$(4)^{\prime}$

$$
\left.\Re Q_{1}^{u}(z), \quad \Re Q_{1}^{\prime}{ }^{u}(z) \quad \text { (not bounded in } R_{1}\right)^{1}
$$

The functions $\left(3_{1}\right)$ clearly contain all the members of $(3)$. Moreover, in the set $\left(3_{1}\right)$ there are certainly some functions not included in (3). In fact, if $R_{1}$ is the region bounded by the curves $B$ and $\tilde{Q}_{x^{0}}^{u}$, for instance, the following is noted.

All the $\Re Q(z)$, corresponding to $\tilde{Q}_{x^{0}}^{u}$ and to those curves $Q_{x^{0}}^{u}$ which (sufficiently far from the origin) are either coincident with or recede to the right of $\tilde{Q}_{x^{0}}^{n}$, will be bounded along $\tilde{Q}_{x^{0}}^{u}$ and, consequently, will be bounded in $R_{1}$. These functions, while not included in (3) will be contained in the set $\left(3_{1}\right)$. Accordingly, there will be fewer members in $\left(4_{1}\right)$ than in (4). A similar fact will be true for the other region $R_{1}$. Consider now a particular region $R_{1}$ and the behaviour, in $R_{1}$, of the members of the corresponding set $\left(4_{1}\right)$.

Suppose that for no $x$, in $R_{1}\left(|x| \geqq \varrho\left(x_{0}\right)\right)$, is a curve $Q_{1 x}^{n}$ situated to the right of a curve $Q_{1 x}^{1 u}(|z| \geqq \varrho(x)) .{ }^{2}$ By a reasoning of the type utilized for a similar purpose in the text immediately following $(4 \mathrm{a})$ the following is proved. For every $x$, in $\dot{R}_{1}$, there exists a curve or curves $C_{x}$, lying in $R_{1}$ and extending to infinity, such that the conditions $I^{\circ}$ and $2^{\circ}$ of the Definition will be satisfied for the members of $\left(3_{1}\right)$ and $\left(4_{1}\right)$.

For some regions $R_{1}$ the condition of the above italicized statement may not hold. Consider such a region $R_{1}$ and the sets $\left(3_{1}\right),\left(4_{1}\right)$ corresponding to it. $R_{1}$ will consist of two overlapping regions, each extending to infinity. Such a pair of regions can be found for every region $R_{1}$ now under consideration. Call any such region $R_{2}$ and, corresponding to a region $R_{2}$, let the $\Re Q(z)$ and the $\Re Q^{\prime}(z)$ be separated into two sets:

$$
\begin{aligned}
& \text { (3⿻) } \quad \Re Q_{2}^{b}(z), \quad \Re Q_{2}^{\prime b}(z) \quad \text { (bounded in } R_{2} \text { ), } \\
& \text { (42) } \quad \Re Q_{2}^{u}(z), \quad \Re Q_{2}^{\prime u}(z) \quad \text { (not bounded in } R_{2} \text { ). }
\end{aligned}
$$

1 The subscripts, here and in the sequel of this proof, should not be confused with the subscripts in ( $\mathrm{I}$ ).

$$
{ }^{2} \text { For } z \text { on } Q_{1 x}^{u} \quad \Re Q_{1}(z)=\Re Q_{1}(x) \text {. }
$$


The set $\left(4_{2}\right)$ will contain fewer members than the set $\left(4_{1}\right)$, corresponding to that one of the regions $R_{1}$ which contains $R_{9}$. This follows by a reasoning of the type employed before in proving that a set $\left(4_{1}\right)$ contained fewer members than a set (4).

We apply the same reasoning to the regions $R_{2}$ as had been applied to the regions $R_{1}$. We are thus led to consequtive subdivisions. Corresponding to the first, second, ...m-th subdivision we get $m$ sets of regions

$$
R_{1}, \quad R_{\mathbf{2}}, \ldots R_{m} .
$$

Associated with a particular region $R_{i}(i=\mathrm{I}, 2, \ldots m)$, belonging to the $i$-th of the above sets of regions, the $\Re Q(z)$ and the $\Re Q^{\prime}(z)$ are divided into two sets:

$$
\begin{array}{lll}
\Re Q_{i}^{b}(z), & \Re Q_{i}^{\prime b}(z) & \text { (bounded in } R_{i} \text { ), } \\
\Re Q_{i}^{u}(z), & \Re Q_{i}^{\prime \prime}(z) & \text { (not bounded in } R_{i} \text { ). }
\end{array}
$$

$A$ set $\left(4_{i}\right)(i=2,3, \ldots m)$ will have fewer members than a set $\left(4_{i-1}\right)$. The regions $R_{i}$ are obtained by subdividing only those of the regions $R_{i-1}$ for which a condition of the type stated in italics, following $(4 \mathrm{a})$, does not hold. The $R_{i-1}$ which are not subdivided are such that $1^{\circ}, 2^{\circ}$ of the Definition will hold for the corresponding sets of functions $\left(3{ }_{i-1}\right),(4 i-1)$. Since there is only a finite number of functions $\Re Q(z), \Re Q^{\prime}(z)$ it is clear that the above process will terminate for $m$ sufficiently great. It follons, accordingly, that $R$ consists of a finite number of overlapping regions

$$
R^{1}, R^{2}, \ldots R^{r_{1}}
$$

each extending to infinity, such that conditions $\mathrm{I}^{\circ}, 2^{\circ}$ of the Definition hold for the functions $\Re Q(z)$ and $\Re Q^{\prime}(z)$, corresponding to curves $Q, Q^{\prime}$ with the limiting direction of $B$ (and $B^{\prime}$ ). These conditions will hold, in general for all functions $\Re Q(z)$ formed with the aid of the set (I). ${ }^{1}$ The curves $C_{x}$ can be so chosen that condition $3^{\circ}$ of the Definition is also satisfied. Thus, in the Case A, either $R$ satisfies $I^{\circ}, 2^{\circ}, 3^{\circ}$ or it consists of a number of overlapping regions each satisfying conditions $\mathrm{I}^{\mathrm{o}}, 2^{\mathrm{o}}, 3^{\mathrm{o}}$.

Case B. The boundaries $B, B^{\prime}$ have different limiting directions. Let $\varepsilon(>0)$ be a small number and let $r_{0}$ and $r_{0}^{\prime}\left(r_{0}>r_{0}^{\prime}\right)$ be the limiting directions of the

1 If a function $\Re Q(z)$ does not vanish along any curve $Q$, possessing the same limiting direction as $B$ (or $B^{\prime}$ ), it increases indefinitely along every $C_{x}\left(x\right.$ and $C_{x}$ in $R$ ), under consideration. 
curves $B$ and $B^{\prime}$, respectively. Let $R_{\varepsilon}$ denote the subregion of $R$ bounded by $B$ and the ray

$$
\arg z=r_{0}-\varepsilon .
$$

Similarly let $R_{\varepsilon}^{\prime}$ denote the subregion of $R$ bounded by the ray

$$
\arg z=r_{0}^{\prime}+\varepsilon \quad\left(r_{0}-\varepsilon>r_{0}^{\prime}+\varepsilon\right) .
$$

The subregion of $R$ bounded by the rays (6) and ( 6 a) will be denoted by $R^{\varepsilon}$. Suppose now that $x$ is in $R_{\varepsilon}$. The curves $Q_{x}$, which correspond to the functions $\Re Q(z)$ vanishing along curves $Q$, each with the same limiting direction as $B$ (and hence situated to the left of $B$ ), will all have the limiting direction of $B$. Along such a curve $\bar{Q}_{x}$ which, sufficiently far from the origin, is on or to the right of all other of these curves the functions $\Re Q(z)$, referred to above, will be all monotone non-decreasing. Thus, curves $C_{x}$ can be chosen, in $R_{\varepsilon}$ and extending from $x$, along which condition $\mathrm{I}^{\circ}$ of the Definition holds for these $\Re Q(z)$. $\quad\left[C_{x}\right.$ will be chosen coincident with or suitably receding to the right from $\bar{Q}_{x}$. Consider the functions $\Re Q(z)$ which do not vanish along any curves with the same limiting direction as $B$. The curves $Q$ along which such a function $\mathfrak{R} Q(z)$ vanishes all have limiting directions distinct from that of any curve $C_{x}$, specified above. On the other hand, every such $\Re Q(z)$ is positive in $R$. Hence these functions are all monotone increasing along $C_{x}$ (for a suitable choice of $C_{x}$ ).

By a similar reasoning we obtain an analogous result for $x$ in $R_{\varepsilon}^{\prime}$.

When $x$ is in $R^{\varepsilon}\left(|x| \geqq \varrho_{\varepsilon}\right)$ every $\Re Q(z)$ is seen to be monotonically increasing along a curve $C_{x}$, consisting of the ray

$$
\arg z=\arg x \text {. }
$$

All conditions of the Definition will be satisfied throughout $R^{\varepsilon}$. The $C_{x}$ can be so chosen that the Definition will be satisfied throughout $R$. In fact, condition $4^{\circ}$. is seen to be satisfied throughout $R$ because along the chosen curves $C_{x}$ all the functions $\Re Q(z), \Re Q^{\prime}(z)$ are monotone beginning from $x$. In other words, $I^{\circ}$ is satisfied with $z(x)=|x|$. Under these circumstances there is no occasion to consider $4^{\circ}$. In the Case $\mathrm{B}$, then, $R$ itself is seen to be proper.

Returning to the Case $A$ we shall now demonstrate that any region, say $R^{k}$, of the set (5) can be separated into a finite number of adjacent proper regions $R_{j}^{k}\left(j=\mathrm{I}, 2, \ldots j_{k}\right)$. Consider first the functions

24-33617. Acta mathematica. 62. Imprimé le 7 novembre 1933. 
each vanishing along a curve $Q$ (or $Q^{\prime}$ ) with the limiting direction at infinity of a boundary of $R^{k}$. For these functions $\mathrm{I}^{\circ}$ is satisfied in $R^{k}$. Let $\Re Q(z)$ be a function of the set $\Re Q_{i}^{u}(z)$ (of (9)) and let $\Re Q^{*}(z)$ be another function of the same set:

$$
\mathcal{H} Q(z)=q(\varrho, \theta)=\sum_{v=0}^{l-1}\left|q_{v}\right| \varrho^{l-v} \cos \left[q_{v}+\frac{l-v}{k} \theta\right] \quad\left(\left|q_{0}\right| \neq 0\right)
$$

$$
\Re Q^{*}(z)=q^{*}(\varrho, \theta)=\sum_{v=0}^{l *-1}\left|q_{v}^{*}\right| \varrho^{l^{*}-v} \cos \left[\dddot{q}_{v}^{*}+\frac{l^{*}-v}{k^{*} \theta}\right] \quad\left(\left|q_{0}^{*}\right| \neq 0\right) .
$$

The totality of curves $Q_{x}$, associated with (9), is specified by the equation

$$
d q(\varrho, \theta) \equiv \frac{\partial}{\partial \varrho} q(\varrho, \theta) d \varrho+\frac{\partial}{\partial \theta} q(\varrho, \theta) d \theta=0
$$

while the curves $Q_{x}^{*}$, corresponding to $(9$ a), are characterized by the relation

$$
d q^{*}(\varrho, \theta) \equiv \frac{\partial}{\partial \varrho} q^{*}(\varrho, \theta) d \varrho+\frac{\partial}{\partial \theta} q^{*}(\varrho, \theta) d \theta=0
$$

Let $\varphi^{*}(r, \alpha)$ denote the angle at which a curve ${ }^{1} Q_{x}$ intersects a curve $Q_{: x}^{*}$ $\left(x=r e^{r-1^{\alpha}}\right)$. From (IO) and (IO a) it follows that

$$
\operatorname{tg} \varphi^{*}(r, \alpha)=\frac{r \frac{\partial}{\partial r} q^{*}(r, \alpha) \frac{\partial}{\partial \alpha} q(r, \alpha)-r \frac{\partial}{\partial r} q(r, \alpha) \frac{\partial}{\partial \alpha} q^{*}(r, \alpha)}{\frac{\partial}{\partial \alpha} q(r, \alpha) \frac{\partial}{\partial \alpha} q^{*}(r, \alpha)+r^{2} \frac{\partial}{\partial r^{*}} q(r, \alpha) \frac{\partial}{\partial r} q^{*}(r, \alpha)}
$$

With the aid of (9) and (9 a) $\operatorname{tg} \varphi(r, \alpha)$ can be expressed in the form

$$
\operatorname{tg} \varphi^{*}(r, \alpha)=\frac{a(r, \alpha)}{b(r, \alpha)}
$$

$$
a(r, \alpha)=-\sum_{v=0}^{l^{*}-1} \frac{l^{k}-v}{k}\left|q_{v}^{*}\right| r^{-\frac{v}{k}} \cos \left(q_{v}^{*}+\frac{l^{k}-v}{k} \alpha\right) \sum_{v=0}^{l-1} \frac{l-v}{k}\left|q_{v}\right| r^{-\frac{v}{k}} \sin \left(\bar{q}_{v}+\frac{l-v}{k} \alpha\right)+
$$

$$
+\sum_{v=0}^{l-1} \frac{l-\nu}{k}\left|q_{v}\right| r^{-\frac{v}{k}} \cos \left(q_{v}+\frac{l-v}{k} \alpha\right) \sum_{\nu=0}^{l^{*}-1} \frac{l^{*}-v}{k}\left|q_{v}^{*}\right| v^{-\frac{\nu}{k}} \sin \left(\bar{q}_{v}^{*}+\frac{l^{*}-\nu}{k} \alpha\right),
$$

${ }^{1}$ We take the unique curves $Q_{x}, Q_{x}^{*}$ possessing the limiting directions of a boundary of $R^{k}$. 


$$
\begin{aligned}
b(r, \alpha)= & \sum_{v=0}^{l} \frac{l-v}{k}\left|q_{v}\right| r^{-\frac{v}{k}} \sin \left(\bar{q}_{v}+\frac{l-v}{k} \alpha\right) \sum_{v=0}^{l *-1} \frac{l^{*}-v}{k}\left|q_{v}^{*}\right| r^{-\frac{v}{k}} \sin \left(\bar{q}_{v}^{*}+\frac{l^{*}-v}{k} \alpha\right)+ \\
& +\sum_{v=0}^{l-1} \frac{l-v}{k}\left|q_{v}\right| r^{-\frac{v}{k}} \cos \left(q_{v}+\frac{l-v}{k} \alpha\right) \sum_{v=0}^{l *-v} \frac{l^{*}-v}{k}\left|q_{v}^{*}\right| r^{-\frac{v}{k}} \cos \left(\bar{q}_{v}^{*}+\frac{l^{*}-v}{k} \alpha\right) .
\end{aligned}
$$

Now

$$
b(r, \alpha)=b(\alpha)+\varepsilon(r, \alpha)
$$

$$
b(\alpha)=\frac{l l^{*}}{k^{2}}\left|q_{0} q_{0}^{*}\right| \sin \left(\bar{q}_{0}+\frac{l}{k} \alpha\right) \sin \left(\bar{q}_{0}^{*}+\frac{l^{*}}{k} \alpha\right)
$$

Here $\varepsilon(r, \alpha)$ has the following property. Given any $\varepsilon(>0)$, independent of $r$ and $\alpha$, there exists a number $r_{\varepsilon}(>0)$, independent of $r$ and $\alpha$, such that

$$
|\varepsilon(r, \alpha)|<\varepsilon
$$

for every $x$, in $R^{k}$, for which $r \geqq v_{\varepsilon}$. This fact is a consequence, in part, of the following considerations. Given any $\varepsilon_{1}(>0)$, independent of $r$ and $\alpha$, there exists a number $r_{\varepsilon_{1}}(>0)$, independent of $r$ and $\alpha$, such that

$$
\left|\cos \left(\bar{q}_{0}+\frac{l}{k} \alpha\right)\right|, \quad\left|\cos \left(\bar{q}_{0}+\frac{l^{*}}{k} \alpha\right)\right|<\varepsilon_{1}
$$

for every $x$, in $R^{k}$, with $r \geqq r_{\varepsilon}$. Moreover, in view of (1 3 ), it follows that

$$
\frac{l l^{*}}{k^{2}}\left|q_{0} q_{0}^{*}\right|\left(\mathrm{I}-\varepsilon^{\prime}\right) \leqq|b(\alpha)| \leqq \frac{l l^{*}}{k^{2}}\left|q_{0} q_{0}^{*}\right| \quad\left(\varepsilon^{\prime}>0\right)
$$

where $\varepsilon^{\prime}$ can be made arbitrarily small by taking $\varepsilon_{1}$ sifficiently small. Accordingly, it is clear that, if $R^{k}$ is defined exclusive of the points for which $r<r_{0}\left(r_{0}\right.$, sufficiently great), the function $b(r, \alpha)$ will necessarily maintain its sign throughout $R^{k}$.

If $a(r, \alpha) \equiv \mathrm{o}$, we shall have $\varphi^{*}(r, \alpha) \equiv \mathrm{o}$ so that every $Q_{x}$ (corresponding to (9)) will be coincident with $Q^{\prime}{ }_{x}\left(x\right.$ in $\left.R^{k}\right)$. Suppose that $a(r, \alpha) \neq 0$ and consider the curve $W^{:}$defined by the equation

$$
a(r, \alpha)=\mathrm{o}
$$

and having at infinity the limiting direction of a boundary of $R^{k}$. Such a curve will exist by virtue of the results of the theory of implicit functions and it will be expressible in the form 


$$
\alpha=u_{0}^{*}+w_{1}^{*} r^{-\frac{1}{k}}+w_{2}^{*} r^{-\frac{2}{k}}+\cdots
$$

Consider all possible curves $W^{*}$,

$$
W_{1}^{*}, \quad W_{2}^{*}, \ldots W_{i}^{*}
$$

formed for pairs of functions picked from the set $\mathfrak{R} Q_{i}^{u}(z)$ (of (9)), and also formed for various pairs of functions selected from the set $\Re_{i}^{\prime} Q_{i}^{\prime}(z)$ (of (9)). These curves are specified in a way precisely analogous to that of the definition of $W^{:}$ with the aid of (I 5). If any of the curves ( 16 ) extend to infinity, interior $R^{k}$, we shall have $R^{k}$ divided in a number of adjacent regions $R_{j}^{k}\left(j=\mathrm{I}, 2, \ldots j_{k}\right)$. These regions will be separated by certain ones of the curves ( 16 ) and interior each one of these regions there will be no curve of the set (I6). Consider a particular region $R_{j}^{k}$. For every $x$ in $R_{j}^{k}$ a certain ordering of the curves $Q_{x}^{u}$, corresponding to all the $\Re Q_{i}^{u}(z)$ of the set (8), will be maintained. Similarly, for every $x$ in $R_{j}^{k}$ an ordering of the curves $Q_{x}^{\prime \prime}$, associated with all the $\Re_{i} Q_{i}^{\prime} u(z)$ of $(8)$, will be maintained.

Consider a function $\Re Q(z)$ of the set $\Re^{\prime} Q_{i}^{u}(z)$ (of (8)) and another function $\Re Q^{\prime}(z)$ of the set $\Re Q_{i}^{\prime}(z)$ (of $(8)$ ). $\Re Q(z)$ will be given by an expression of the form (9). On the other hand, is $Q^{\prime}(z)$ will be defined by (9 a) with $Q^{*}, q^{*}$, $l^{*}, \bar{q}^{:}$replaced by $Q^{\prime}, q^{\prime}, l^{\prime}, \bar{q}^{\prime}$ respectively. We specify a curve $W^{\prime}$ just as $W^{*}$ had been previously specified. In connection with this curve all the formulas from (9) to, and including, ( 15 a) will be valid (with the indicated change of notation). To either side of this curve the angle $\varphi^{\prime}(r, \alpha)\left(x=r e^{r^{-1}{ }^{\alpha}}\right)$, defined as the angle at which $Q_{x}$ intersects $Q_{x}^{\prime}$ (at $x$ ), will maintain its sign. If it were possible for a curve $W^{\prime}$ to separate $R^{k}$ into two regions $R^{1, k}$ and $R^{2, k}$, each extending to infinity, it would follow that for every $x$, in $R^{1, k}, \varphi(r, \alpha)$ maintains one sign; while, in $R^{2, k}, \varphi(r, \alpha)$ would maintain the opposite sign. For every $x$ in one of these regions $Q_{x}$ would recede to the left of $Q_{x}^{\prime}$, while the opposite would be true for every $x$ in the other region. This is contrary to the proved. properties of $R^{k}$. Hence a curve $W^{\prime}$ cannot separate $R^{k}$ in the stated manner. Thus, in particular, for every $x$ in $R_{j}^{k}$ every $Q_{x}^{u}$, corresponding to the $\mathfrak{H} Q_{i}^{u}(z)$ of the set (8), will recede to the left of every $Q_{x}^{\prime \prime}$, associated with the $\Re Q_{i}^{\prime \prime}(z)$ of $(8)$. Exceptionally, a $Q_{x}$ may be coincident with a $Q_{x}^{\prime}\left(x\right.$ in $\left.R_{j}^{k}\right)$. The above statement in italics implies that, for any fixed $x$ in $R_{j}^{k}$ (or, more generally, in $R^{k}$ ), the curves $Q_{x}$ and $\dot{Q}_{x}^{\prime}$ do not intersect at any points $z$ distinct from $z=x$. In view of the two preceding italicized statements, curves $C_{x}$ can be always specified so 
that (in addition to $3^{\circ}$ ) the condition $\mathrm{I}^{\circ}$ is satisfied (with $z(x)=|x|$ ) for all the functions of the set (8). ${ }^{1}$ For the set (8), then, there will be no occasion to consider $4^{\circ}$.

We note that every $C_{x}$ is chosen as a regular curve. The values, on $C_{x}$, of a function $\Re Q(z)$ are accordingly representable by a convergent series of the form

$$
\mathfrak{R} Q(z)=\sum_{v=l}^{-\infty} g_{v} \varrho^{\frac{k}{k}}
$$

Hence $\Re Q(z)$ has a limited number of maxima and minima, along $C_{x}$, between $z=x$ and the point $z$ for which $|z|=z(x)$. If $\mathcal{R} Q(z) \leqq M$ in $R_{j}^{k}$, then the corresponding function $h(x)$, as defined in (2), is less than $h=\eta M$, where $\eta$ depends on the number of maxima and minima (referred to above) and is independent of $x$ ( $x$ in $\left.R_{j}^{k}\right)$. Accordingly, $4^{\circ}$ is seen to be satisfied, in $R_{j}^{k}$, for all $\mathfrak{R} Q(z)$ (and $\mathfrak{R} Q^{\prime}(z)$ ) bounded in $R_{j}^{k}$.

The remaining functions $\mathfrak{R} Q(z)$, which do not vanish along any curve possessing the limiting direction of a boundary of $R^{k}$, do not cause any difficulty. Thus the truth of the italicized statement preceding (8) has been made evident. This completes the proof of the Lemma.

4. Formal Integration. In view of the purposes at hand it is essential to solve formally the equation

$$
y^{(1)}(x)=e^{Q(x)} x^{r} x(x)
$$

where the $\sigma$-series (cf. $\$ \mathrm{I}) x(x)$ is given by

$$
\begin{aligned}
& x(x)=x^{0}(x)+x^{\prime}(x) \log x+\cdots+x^{m}(x) \log { }^{m} x \\
& \quad x^{i}(x)=x_{0}^{i}+x_{1}^{i} x^{-\frac{1}{k}}+x_{2}^{i} x^{-\frac{2}{k}}+\cdots \quad(i=0,1, \ldots m)
\end{aligned}
$$

and $Q(x)$ is given by $[(2) ; \S 2]$. It will be supposed that not all the constants $x_{0}^{i}(i=\mathrm{I}, 2, \ldots m)$ are zero. This equation we shall satisfy by a series of the form

$$
y(x)=e^{Q(x)} x^{r+\alpha} \eta(x),
$$

$$
\begin{aligned}
\eta(x)= & \eta^{0}(x)+\eta^{1}(x) \log x+\cdots+\eta^{m+1}(x) \log ^{m+1} x \\
& \eta^{i}(x)=\eta_{0}^{i}+\eta_{1}^{i} x^{-\frac{1}{k}}+\eta_{2}^{i} x^{-\frac{2}{k}}+\cdots \quad(i=0, \mathrm{I}, \ldots m+1)
\end{aligned}
$$

${ }^{1}$ One may select a suitable $Q_{x}$ or $Q_{x}^{\prime}$ curve of the set of curves associated with (8). 
Case I. $\quad Q(x) \neq 0$. Write

(3) $x Q^{(1)}(x)+(r+\alpha)=p(x)=p_{l} x^{\frac{l}{k}}+p_{l-1} x^{\frac{l-1}{k}}+\cdots+p_{1} x^{\frac{1}{k}}+p_{0} \quad\left(p_{l} \neq 0 ; l \geqq \mathrm{I}\right)$.

Substitution of (2) in (r) gives, after division by $e^{Q(x)} x^{r}$,

$$
x^{-a} x \varkappa(x)=p(x) \eta(x)+x \eta^{(1)}(x) .
$$

On arranging the two members of (4) according to powers of $\log x$ comparison of coefficients of $\log ^{\lambda} x$ will give the relations

$$
\begin{aligned}
& x^{-\alpha}\left[x_{0}^{2} x+x_{1}^{\lambda} x^{\frac{k-1}{k}}+x_{2}^{\dot{\lambda}} x^{\frac{k-2}{k}}+\cdots\right]=\left[p_{l} x^{l}+\cdots+p_{0}\right]\left[\eta_{0}^{\lambda}+\eta_{1}^{\lambda} x^{-\frac{1}{k}}+\cdots\right]+ \\
& +\left\{(\lambda+\mathrm{I}) \eta_{0}^{\lambda+1}+\left[(\lambda+\mathrm{I}) \eta_{1}^{\lambda+1}-\frac{1}{k} \eta_{1}^{\lambda}\right] x^{-\frac{1}{k}}+\left[(\lambda+\mathrm{I}) \eta_{2}^{\lambda+1}-{ }_{k}^{2} \eta_{2}^{\lambda}\right] x^{-\frac{2}{k}}+\cdots\right\} \\
& (\lambda=\mathrm{o}, \mathrm{I}, 2, \ldots m)
\end{aligned}
$$

and

(4 b) $\mathrm{o}=\left[p_{l} x^{\frac{l}{k}}+\cdots+p_{0}\right]\left[\eta_{0}^{m+1}+\eta_{1}^{m+1} x^{-\frac{1}{k}}+\cdots\right]-\left[\frac{1}{k} \eta_{1}^{m+1} x^{-\frac{1}{k}}+\frac{2}{k} \eta_{z}^{m+1} x^{-\frac{2}{k}}+\cdots\right]$.

Since $p_{l} \neq 0$ from $(4 \mathrm{~b})$ it follows that necessarily

$$
\eta_{i}^{m+1}=0 \quad(i=0, \mathrm{I}, 2, \ldots)
$$

Consider now (4a) with $\lambda=m$. Comparison of the highest powers of $x$ gives the value of $\alpha$,

$$
\alpha=\frac{k-l}{k}
$$

This value is substituted in $p_{0}$ in (3). Comparison of the coefficients of

determines uniquely

$$
x^{\frac{l}{k}}, x^{\frac{l-1}{k}}, \ldots x^{\frac{1}{k}}
$$

$$
\eta_{0}^{m}, \quad \eta_{1}^{m}, \ldots \eta_{l-1}^{m}
$$

with the aid of the equations

$$
\begin{gathered}
\varkappa_{j}^{m}=\eta_{j}^{m} p_{l}+\eta_{j-1}^{m} p_{l-1}+\cdots+\eta_{0}^{m} p_{l-j} \\
(j=\mathrm{o}, \mathrm{I}, \ldots l-\mathrm{I}) .
\end{gathered}
$$


On the other hand, comparing the coefficients of $x^{-\frac{v}{p}}(v=0,1, \ldots)$ we get

$$
\begin{gathered}
x_{k+v}^{m}=\left[\eta_{l+v}^{m} p_{l}+\cdots+\eta_{v}^{m} p_{0}\right]+(m+\mathrm{I}) \eta_{v}^{m+1}-\frac{\nu}{k} \eta_{v}^{m} \\
(v=0, \mathrm{I}, \ldots)
\end{gathered}
$$

These equations determine in succession and uniquely the constants

$$
\eta_{l+\nu}^{m} \quad(\nu=0, \mathrm{I}, \ldots)
$$

Next the coefficients of $\eta^{m-1}(x)$ may be determined uniquely by means of sets of equations of the type of $(5 \mathrm{~b})$ and $(5 \mathrm{c})$, derived by comparison of the coefficients of the various powers of $x$ from $(4 \mathrm{a} ; \lambda=m-1)$. Suppose the formal series

$$
\eta^{m+1}(x), \quad \eta^{m}(x), \quad \eta^{m-1}(x), \ldots \eta^{\sigma+1}(x)
$$

have been determined (by means of consecutive applications of $(4 \mathrm{~b})$ and of $(4 \mathrm{a} ; \lambda=m, m-\mathrm{I}, \ldots \sigma+\mathrm{I})$. The series $\eta^{\sigma}(x)$ can then be determined from $(4 \mathrm{a} ; \lambda=\sigma)$. In fact, comparison of the coefficients of $x^{\frac{l}{k}}, \ldots, x^{\frac{1}{k}}$ will yield the equations

$$
\begin{gathered}
\varkappa_{j}^{\sigma}=\eta_{j}^{o} p_{l}+\eta_{j-1}^{\sigma} p_{l-1}+\cdots+\eta_{0}^{\sigma} p_{l-j} \\
(j=0, \mathrm{I}, \ldots l-\mathrm{I}) .
\end{gathered}
$$

Equating the coefficients of the other powers of $x$ we obtain

$$
\begin{gathered}
x_{l+v}^{\sigma}=\left[\eta_{l+v}^{\sigma} p_{l}+\cdots+\eta_{\nu}^{\sigma} p_{0}\right]+(\sigma+\mathrm{I}) \eta_{\nu}^{\sigma+1}-\frac{\nu}{k} \eta_{\nu}^{\sigma} \\
(v=0, \mathrm{I}, \ldots)
\end{gathered}
$$

With the aid of $(6)$ and $\left(6\right.$ a) the series $\eta^{\sigma}(x)$ can be determined (in terms of the coefficients of $\left.\eta^{\sigma+1}(x)\right)$. Thus, in the case under consideration consequtive application of (5), (6) and (6 a) for $\sigma=m, m-1, \ldots$, o will serve to determine a formal series solution of the form (2), (2 a) (with $\alpha=\frac{k-l}{k}$ ). 
Case II. $Q(x) \equiv 0 . \quad$ In place of (4) we obtain

$$
x^{-\alpha} x \times(x)=(r+\alpha) r_{i}(x)+x \eta^{(1)}(x) .
$$

Comparison of the coefficients of the various powers of $\log x$ will give

$$
\begin{aligned}
& \text { (7 a) } x^{1-\alpha}\left[x_{0}^{\lambda}+x_{1}^{\lambda} x^{-\frac{1}{k}}+\cdots\right]=(r+\alpha)\left[\eta_{0}^{\lambda}+\eta_{1}^{\lambda} x^{-\frac{1}{k}}+\cdots\right]+ \\
& +\left\{(\lambda+\mathrm{I}) \eta_{0}^{\lambda+1}+\left[(\lambda+\mathrm{I}) \eta_{i}^{\lambda+1}-\frac{\mathrm{I}}{k} \eta_{\mathrm{i}}^{\lambda}\right] x^{-1} k+\left[(\lambda+\mathrm{I}) \eta_{i}^{\lambda+1}-\frac{2}{k} \eta_{2}^{\lambda}\right] x^{-\frac{2}{k}}+\cdots\right\} \\
& (2=0,1,2, \ldots m)
\end{aligned}
$$

and

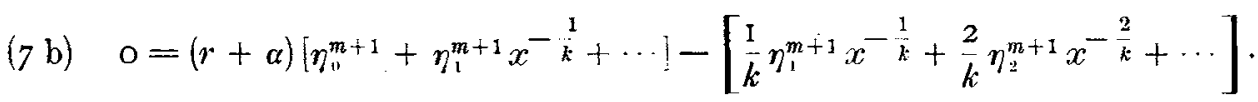

Comparison of the highest powers of $x$ in $(7$ a) leads us to take $\alpha=1$. Substitute $\alpha=\mathrm{I}$ in $(7 \mathrm{a})$ and $(7 \mathrm{~b})$.

Suppose first that

$$
r+\mathrm{I} \neq \frac{v}{k} \quad(v=0, \mathrm{I}, 2, \ldots)
$$

From $(7 \mathrm{~b})$ it will follow that

$$
\left(r+\alpha-\frac{v}{k}\right) \eta_{r}^{m+1}=0 \quad(v=0, \mathrm{I}, 2, \ldots)
$$

that is, $\eta^{m+1}(x)$ will be formally zero. The series $\eta^{\eta}(x), \eta^{m-1}(x), \ldots \eta^{0}(x)$ will be then determined in succession by means of the sets of equations $(6$ a) formed for $\sigma=m, m-\mathrm{I}, \ldots, \mathrm{o}$; here we let $l=0$ and $p_{0}=r+\mathrm{I}$.

It remains to consider the case

$$
r+\mathbf{I}=\frac{s}{k} \quad \text { (integer } s \geqq 0 \text { ). }
$$

The equations $(8 \mathrm{a})$ will leave $\eta_{s}^{m+1}$ undefined but all the other coefficients in $\eta^{m+1}(x)$ will necessary be all zero. Thus

$$
\eta^{m+1}(x)=\eta_{s}^{m+1} x^{-\frac{k}{k}}
$$

Let $\left(\sigma^{\prime}\right.$ a) denote the equations $\left(6\right.$ a) formed with $l=0$ and $p_{0}=r+1$. The set of equations $\left(6^{\prime} \mathrm{a} ; \sigma=m\right)$, 


$$
x_{v}^{m}=\left(v+\mathrm{I}-\frac{v}{k}\right) \eta_{v}^{m}+(m+\mathrm{I}) \eta_{v}^{m+1} \quad(v=0, \mathrm{I}, \ldots)
$$

determines uniquely the constants $\eta_{v}^{m}(v=0, \mathrm{I}, \ldots s-\mathrm{I}, s+\mathrm{I}, \ldots)$. On the other hand, (IO; $\nu=s$ )

$$
x_{s}^{m}=0 \eta_{s}^{m}+(m+\mathrm{I}) \eta_{s}^{m+1}
$$

determines uniquely the constant $\eta_{s}^{m+1}$ (which hitherto was left undefined); however, equations (IO) will leave $\eta_{s}^{m}$ undefined. Suppose now that successive use of the sets of equations $\left(\sigma^{\prime}\right.$ a) (with $\sigma=m, \sigma=m-\mathrm{I}, \ldots \sigma=\lambda+\mathrm{I}$ ) enabled us to determine uniquely all the coefficients in the series

$$
\eta^{m+1}(x), \eta^{m}(x), \eta^{m-1}(x), \ldots \eta^{i+1}(x),
$$

except $\eta_{s}^{\lambda+1}$, which is left undefined. Application of $\left(6^{\prime}\right.$ a $\left.; \sigma=\lambda\right)$ will determine uniquely $\eta_{s}^{\lambda+1}$ and all the coefficients $\eta_{v}^{\lambda}(\nu=0, \mathrm{I}, \ldots)$, except $\eta_{s}^{\lambda}$. Thus (9 a) and the sets of equations $\left(6^{\prime} \mathrm{a}\right)$, formed for $\sigma=m, m-\mathrm{I}, \ldots \mathrm{I}, \mathrm{o}$, will serve to determine uniquely the series

$$
\eta^{m+1}(x), \eta^{m}(x), \ldots \eta^{0}(x)
$$

except that the coefficient $\eta_{*}^{0}$ will be arbitrary.

Lemma 2. Every formal equation (I), (I a) possesses a formal solution of the type (2), (2 a). When $Q(x) \neq 0$, so that

$$
Q(x)=\sum_{v=0}^{v=l-1} q_{v} x^{l-v v} \quad\left(q_{v} \neq 0 ; l \geqq 1\right)
$$

in the solution (2), (2 a) we have

$$
a=\frac{k-l}{k}
$$

while $\eta^{m+1}(x)=0$ and the series $\eta^{m}(x), \ldots \eta^{0}(x)$ are determined in succession with the aid of $(6),(6 \mathrm{a})(\sigma=\dot{m}, m-\mathrm{I}, \ldots \mathrm{o})$. When $Q(x) \equiv \mathrm{o}$ and $r+\mathrm{I} \neq \frac{v}{k}$ $(v=0, \mathrm{I}, 2, \ldots)$ we have $\alpha=\mathrm{I}, \eta^{m+1}(x)=0$, while the series $\eta^{m}(x), \eta^{m-1}(x), \ldots \eta^{0}(x)$ are determined in succession by the sets ( 6 a) (formed for $\sigma=m, \ldots \circ$ with $l=0$ and $\left.p_{0}=r+\mathrm{I}\right)$. When $Q(x) \equiv \mathrm{o}$ and for some integer $s(\geqq 0) r+\mathrm{I}=\frac{s}{k}$, we have 25-33617. Acta mathematica. 62. Imprimé le 7 novembre 1933. 
$a=\mathrm{I}$ and $\eta^{m+1}(x)$ will be of the form (9 a). In this case the coefficients in $\eta^{m+1}(x)$, $\eta^{m}(x), \ldots \eta^{0}(x)$ are all uniquely determined by $\left(\sigma^{\prime}\right.$ a), formed for $\sigma=m, m-\mathbf{I}, \ldots$, except that $\eta_{8}^{0}$ will be arbitrary.

5. Analytic Integration. Let the function

$$
H(x)=e^{Q(x)} x^{r} h(x)
$$

where $Q(x)$ is a polynomial in $x^{1}$ (unless $Q(x) \equiv 0$ ), be analytic in a neighborhood $R$ of infinity. We shall say that $H(x)$ is asymptotic in $R$ to $e^{\mathcal{Q}(x)} x^{r} x(x)[x(x)$ of the form ( $1 \mathrm{a} ; \$ 4)]$,

$$
H(x) \sim e^{Q(x)} x^{r} x(x)
$$

to $w$ terms provided that

$$
h(x)=h^{0}(x)+h^{1}(x) \log x+\cdots+h^{m}(x) \log { }^{m} x
$$

where, for $i=\mathrm{o}, \mathrm{I}, \ldots m$

$$
\begin{gathered}
h^{i}(x)=x_{0}^{i}+x_{1}^{i} x^{-\frac{1}{k}}+\cdots+x_{u-1}^{i} x^{-\left(\frac{u-1}{k}\right)}+b_{w}^{i}(x) x^{-\frac{u t}{k}} \\
\left(\left|b_{w}^{i}(x)\right| \leqq b_{w}^{i} ; x \text { in } R\right) .
\end{gathered}
$$

If the relations $(2 \mathrm{~b})$ hold for every positive integer $u$ so that every constant $b_{w}^{i}(i=\mathrm{o}, \mathrm{I}, \ldots m ; w=\mathrm{I}, 2, \ldots)$ is finite the asymptotic relationship (2) is of course in the ordinary sense (that is, to infinity of terms).

Consider now the analytic equation in $y$,

$$
y^{(1)}(x)=e^{Q(x)} x^{r} h(x),
$$

where the second member is a known function, analytic in $R$ and satisfying in $R$ the asymptotic relationship (2) to $w$ terms ( $w$ suitably great). The region $R$ will be specified more definitely as follows. It is to consist of the part of the complex plane for which $r \geqq \varrho_{1}\left(\varrho_{1}\right.$ suitably great) and it will be bounded by two regular curves

$$
B_{l}, B_{r}
$$

Furthermore, none of the $Q$ curves satisfying the equation

$$
\mathfrak{R} Q(x)=\mathrm{o}
$$


will be supposed to be interior $R$. Either one or both curves $B_{l}, B_{r}$ may be $Q$ curves associated with the equation (4). Finally, it is assumed that for $x$ in $R$

$$
\Re Q(x) \geqq 0 .
$$

By Lemma $2(\$, 4)$ the formal equation

$$
y^{(1)}(x)=e^{Q(x)} x^{r} x(x),
$$

corresponding to (3), has a formal solution

$$
s(x)=e^{Q(x)} x^{r+\alpha} \eta(x)
$$

of the form $(2),(2$ a) $(\S 4)$. Let

$$
t(x)=e^{Q(x)} x^{r+\alpha} \Gamma(x)
$$

denote $s(x)$ with the terms containing powers of $x$

omitted. Substitute in (3)

$$
x^{\frac{t+v}{k}} \quad(\nu=0,1,2, \ldots ; t<w)
$$

$$
y(x)=t(x)+z(x) .
$$

The new variable $z(x)$ satisfies the equation

$$
\begin{aligned}
& z^{(1)}(x)=e^{Q(x)} x^{r} \sum_{i=0}^{m}\left[x_{0}^{i}+x_{1}^{i} x^{-\frac{1}{k}}+\cdots+x_{u-1}^{i} x^{-\left(\frac{w-1}{k}\right)}\right. \\
& +b_{w}^{i}(x) x^{-\frac{w}{k}} \log ^{i} x-\frac{d}{d x}\left(e^{Q(x)} x^{r+\alpha} \sum_{i=0}^{m+1}\left[\eta_{0}^{i}+\eta_{1}^{i} x^{-\frac{1}{k}}+\cdots+\eta_{t-1}^{i} x^{-\left(\frac{t-1}{k}\right)}\right] \log ^{i} x\right) .
\end{aligned}
$$

Taking account of the relations satisfied by the constants involved in the series of the last members in (6) and $(6$ a) (cf. $\$$ 4), arranging the second members of (8) according to powers of $\log x$ and arranging the coefficients of these powers as power series in $x^{-\frac{1}{k}}$, it is observed that

$$
z^{(1)}(x)=e^{Q(x)} x^{r} b_{\Gamma}(x) x^{-\frac{\Gamma}{k}}
$$

Here $\Gamma=\Gamma(w)$ is an integer $(<t)$ and $b_{\Gamma}(x)$ is a function, analytic in $R(|x| \neq \infty)$, such that to a few terms 


$$
b_{\Gamma}(x) \sim \beta_{\Gamma}(x)
$$

$(x$ in $R)$

where $\beta_{\Gamma}(x)$ is a $\sigma$-series with not all the constant terms in the power series factors zero. It is of importance to note that, if it were possible to take $w$ arbitrarily great, $\Gamma$ can be so chosen that $\lim _{w=\infty} \Gamma(w)=\infty$. The precise relationship between $\boldsymbol{r}$ and $w$ is not essential for the purposes of this paper.

A. Suppose $Q(x) \neq 0$ and $R$ is part of a region $\bar{R}(Q)$ (cf. $\S 2$ ). We take then

$$
z(x)=\int_{x_{n}}^{x} e^{Q(z)} z^{r} b_{r}(z) z^{-I} k d z
$$

where the path of integration lies in $R$ and is from a fixed point $x_{0}$ (on the boundary of $R$ and, say, nearest to $x=0$ ). There exists a constant $b$, depending only on the shape of the region $R$, such that the length of the path is $<b|x|$. Since $\left|e^{Q(x)}\right|$ will increase, as $|x| \rightarrow \infty$ in $R$, more rapidly than any power of $|x|$ it follows that, depending on $\Gamma$, there exists a constant $\varrho_{\Gamma}$ such that, for $|x| \geqslant \varrho_{\Gamma}$, the integrand attains its maximum absolute value at $x$. Thus

$$
|z(x)|<b \mid x \| e^{Q(x)} x^{r} b_{\Gamma}(x) x^{-\frac{r}{k} \mid} \quad\left(|x| \geqq \varrho_{\Gamma} ; x \text { in } R\right)
$$

Now $\left|b_{\Gamma}(x)\right|$ cannot increase faster than $|\log x|^{m+1}$; on the other hand, for $|x|<\varrho_{\Gamma}$

$$
|z(x)| \leqq c_{I}
$$

$(x$ in $R)$.

Hence from (9 a) and (9 b) it follows that

$$
z(x)=e^{Q(x)} x^{r} z_{\Gamma^{\prime}}(x) x^{-\left(\frac{I-k-1}{k}\right)}
$$

where

(IO a)

$$
\left|z_{\Gamma}(x)\right| \leqq d_{\Gamma}
$$
$(x$ in $R)$.

The number $d_{\Gamma}$ could be selected as the upper bound of the following two functions

$$
\begin{array}{cl}
b \mid b_{\Gamma}(x) x^{-\frac{1}{k} \mid} & \left(|x| \geqq \varrho_{\Gamma} ; x \text { in } R\right), \\
c_{\Gamma}\left|e^{-Q(x)} x^{-r} x^{\Gamma-k-1}\right| & \left(|x| \leqq \varrho_{\Gamma} ; x \text { in } R\right) .
\end{array}
$$

On substituting (IO) in (7) it is found that 


$$
y(x) \sim s(x)
$$

$(x$ in $R)$

(cf. (6 a)) to $\Gamma^{\prime}=\Gamma^{\prime}(w)$ terms. If it were possible to let $w \rightarrow \infty, \lim _{w} \Gamma^{\prime}(w)=\infty$.

B. Let $\Re Q(x) \neq 0$. Suppose that a region $\vec{R}(Q)$ exists such that one of the two curves $B_{l}, B_{r}$, say $B_{r}$, is in $\bar{R}(Q)$. Suppose that no region $\bar{R}(Q)$ exists such that the other one of these curves is in $\overparen{R}(Q)$. In this case, then, $(17 ; \S 2)$ could not hold along $B_{l}$ for every $\beta$. Hence it can be seen without difficulty that for some $\beta=\beta_{1}$ the left member in $(17 ; \S 2)$ approaches zero along $B_{l}$. We note that with $B_{l}$ there will be associated a curve $Q^{*}$ (cf. $\S 2$ ) with a limiting direction different from that of $B$.

We define $z(x)$ by the integral

$$
\begin{gathered}
z(x)=\int_{\infty}^{x} e^{Q(z)} z^{r} b_{\Gamma}(z) z^{-\frac{\Gamma}{k}} d z \\
\left(\frac{\Gamma}{k}>\beta_{1}, \quad \text { sufficiently great }\right) .
\end{gathered}
$$

The path of integration will be taken in $R$ and will extend from infinity along $B_{l}$. When $x$ is in $R$ between (or on) the curves $B_{l}, Q^{*}$ the path of integration in (12) will be deformed as follows:

$$
\int_{\infty}^{x^{\prime}}+\int_{x^{\prime}}^{x}
$$

Here the first integral is along $B_{l}$ from infinity to the point $x^{\prime}$, on $B_{l}$, such that $\left|x^{\prime}\right|=|x|=r$. The second integral is along the circular arc, with its center at the origin, extending from $x^{\prime}$ to $x$. Along the first path

$$
\left|e^{Q(z)} z^{-\frac{\Gamma}{k}}\right|
$$

attains its maximum at $x^{\prime}$. In view of the properties of curves $Q^{*}$, established in $\$ 2$, the function

$$
\left|e^{Q(z)} \bar{z}^{-\frac{r^{r}}{k}}\right|=r^{-\frac{r}{k}}\left|e^{Q(z)}\right| \quad(r \text { fixed })
$$

will increase monotonically as $z$ varies from $x^{\prime}$ to $x$ along the second path. Generally speaking, the function ( 12 b) will attain its maximum, along the com- 
bined path of ( 12 a), at $x$. By a reasoning analogous to that employed in deriving (10) it is again proved that, when $z(x)$ is expressed in the form (10), an inequality similar to ( 10 a) will hold for $x$ in $R$ between (or on) $B_{l}$ and $Q^{*}$; in the corresponding region the relation (I I) will also hold. Consider now, if there is an occasion to do so, the properties of the integral (12) when $x$ is in $R$ between $Q^{*}$ and $B_{r}$. In this case the integral will be deformed as follows:

$$
\int_{x}^{x_{0}}+\int_{x_{0}}^{x}
$$

where $x_{0}$ is the fixed point in the finite part of the plane common to $Q^{*}$ and the boundary of $R$. The first integral is along $B_{l}$, while the second integral is along a path extending within the closed subregion of $R$, bounded by $B_{l}$ and $Q^{*}$. Now $B_{l}$ and $Q^{*}$ have different limiting directions at infinity (cf. $\$ 2$ ) and along $B_{r}\left|x^{-\beta}\right| e^{\Re Q(x)} \rightarrow \infty$ for every $\beta(>0)$. Hence, in the case under consideration, $\left|e^{Q(x)}\right|$ increases sufficiently rapidly (as $|x| \rightarrow \infty$ ) to enable precisely the same treatment of the first integral ( 13 ) as had been applied to the integral (9). On using (7) it will follow that

$$
y(x)-c_{0} \sim s(x) \quad\left(x \text { between } Q^{*} \text { and } B_{r}\right)
$$

to $\Gamma^{\prime \prime}=I^{\prime \prime}(w)$ terms $\left[\lim _{w} \Gamma^{\prime \prime}=\infty\right.$, if can let $\left.w \rightarrow \infty\right]$, where the constant $c_{n}$ is defined by the first integral ( ${ }^{3} 3$ ). But in view of the behaviour of $\Re Q(x)$ in the region under consideration it is observed that $c_{9} \sim 0$ (in the ordinary sense). Thus an asymptotic relation of the type of (I I) will continue to remain valid throughout $R$ in the case under consideration.

$\mathrm{C}$ Suppose now that $\Re Q(x) \neq 0$, while every region $R(Q)$ contains neither $\boldsymbol{B}_{l}$ nor $\boldsymbol{B}_{r}$. Assume, moreover, that $\boldsymbol{B}_{l}$ and $\boldsymbol{B}_{r}$ have different limiting directions. In this case the integral ( 12 ), or an analogous integral with the path of integration along $B_{r}$, can be used. Let $Q_{l}^{*}$ be the curve associated with $B_{l}$. Necessarily $Q_{i}^{*}$ will lie between $B_{l}$ and $B_{r}$, its limiting direction being distinct from those of $B_{l}$ and $B_{r}$ (cf. $\S 2$ ). For some $\beta^{\prime}$ we shall have

$$
\lim _{|x|}\left|x^{-\beta^{\prime}}\right| e^{\Re Q(x)}=0,
$$

as $|x| \rightarrow \infty$ along both $B_{l}$ and $B_{r}$. Let $R^{l}$ denote a subregion of $R$, bounded on one side by $B_{l}$ and on the other side by a curve $\bar{B}_{r}$ with a limiting direction at 
infinity and lying in a region $\bar{R}(Q) .{ }^{1} \quad$ Similarly, define a region $R^{r}$ with boundaries $B_{r}$ and $\bar{B}_{l}$. The regions $R^{l}$ and $R$ will have in common a region bounded by $\vec{B}_{l}$ and $\bar{B}_{r}$ (the latter two curves having different limiting directions).

It is observed that for $x$ in $R^{l}$ the integral (12) can be treated precisely as in the preceding case. Thus, a solution $y(x)$, satisfying an asymptotic relation of the type of (I I) (for $x$ in $R^{l}$ ) will exist. Call this solution $y_{l}(x)$. Let $y_{r}(x)$ denote the analogous solution satisfying the asymptotic relation (I I) for $x$ in $R^{r}$,

$$
y_{l}(x)=y_{r}(x)+c .
$$

Thus there exists a constant a such that, to $\Gamma_{1}(<w)$ terms,

$$
y_{l}(x)-c \sim s(x)
$$

for $x$ in $R^{r}$. The constant $c$ cannot be discarded in this asymptotic relationship.

D. Let it be assumed that $\Re Q(x) \neq \equiv$; suppose, moreover, that $B_{r}$ and $B_{l}$ have same limiting directions at infinity, while no region $\bar{R}(Q)$ contains either $B_{r}$ or $B_{l}$. Then, depending on the position of the $Q$ curves along which $\Re Q(x)=0$, $\Re Q(x)$ will be monotonically increasing as $x$ moves along the circular arc

$$
|x|=\varrho \quad\left(\geqq \varrho_{0}>0\right)
$$

from $B_{l}$ to $B_{r}$ (or from $B_{r}$ to $B_{l}$, as the case may be). ' It is clear that integrals of type ( 12 a) can be used yielding an asymptotic relation of type (I I), valid for $x$ in $R$.

E. Consider the remaining case when $\Re Q(x) \equiv 0$. Define $z(x)$ by means of the integral

$$
z(x)=\int_{\infty}^{x} e^{Q(z)} z^{r} b_{\Gamma}(z) z^{-\frac{\Gamma}{k}} d z
$$

where the path of integration lies in $R$ and extends from infinity. We have

$$
\begin{aligned}
|z(x)| & \leqq b_{r}^{\prime} \int_{\infty}^{x}\left|z^{-\frac{r^{r}}{k}+\frac{1}{k}+r} d z\right| \\
& <b_{\Gamma}^{\prime \prime}|x|^{-\frac{T^{\prime}}{k}+\frac{1}{k}+r+1}
\end{aligned}
$$
$(x$ in $R)$

\footnotetext{
${ }^{1} \bar{B}_{r}$ can always be chosen, in $R$, with the same limiting direction at infinity as that of $B_{r}$.
} 
provided, of course, $\Gamma$ is sufficiently great. A solution $y(x)=t(x)+z(x)$ will evidently exist such that a relation of the type of (II) is satisfied.

Consider the equation (3) with the second member satisfying, for $x$ in $R$, the asymptotic relationship (2) in the ordinary sense. Let $y_{0}(x)$ denote a solution, as obtained before, such that

$$
y_{0}(x) \sim s(x)
$$

to $\Gamma\left(w_{0}\right)$ terms; this solution being obtained for some sufficiently great value of $w=w_{0}$. Let $y_{1}(x)$ denote another solution, corresponding to a greater value of $w=w_{1}\left(>w_{0}\right)$,

$$
y_{1}(x) \sim s(x)
$$

(to $\Gamma\left(w_{1}\right)$ terms),

'Taking $w_{1}$ sufficiently great, $\Gamma\left(w_{1}\right)>\Gamma^{*}(w)$; in fact, $\lim _{w} \Gamma(w)=\infty$. Let $t_{0}(x)$ and $t_{1}(x)$ be the corresponding functions $t(x)\left(t_{0}<t_{1}\right)$. On writing

$$
y_{0}(x)-y_{1}(x)=c_{0,1}
$$

it is seen that

$$
\begin{aligned}
c_{0,1} & =t_{0}(x)-t_{1}(x)+\int^{x}\left\{\left[H(z)-\frac{d}{d z} t_{0}(z)\right]\right. \\
& \left.-\left[H(z)-\frac{d}{d z} t_{1}(z)\right]\right\} d z \\
& =\lim \left[t_{1}(z)-t_{0}(z)\right] .
\end{aligned}
$$

In the case $A$ the limit is taken as approaches $x_{0}$. In this case, in general, $c_{0, I} \neq 0$. In view of (I 8$)$ and in view of the fact that $R Q(x) \rightarrow \infty$ (as $|x| \rightarrow \infty$ in $R$ ) sufficiently rapidly it is seen that

$$
y_{0}(x) \sim s(x)
$$$$
(x \text { in } R)
$$

to $\Gamma\left(w_{1}\right)$ terms. But $w_{1}$ and hence $\Gamma\left(w_{1}\right)$ can be made arbitrarily great. Thus (19) will hold in the ordinary sense.

In the case $B$, with say $B_{l}$ not in any region $R(Q)$, the limit in ( 18 a) is taken as $z$ approaches infinity along $B_{l}$. Thus, we shall have $c_{0,1}=\mathrm{O}^{1}$ so that

$$
y_{0}(x)=y_{1}(x) \sim s(x) \quad(x \text { in } R)
$$

\footnotetext{
${ }^{1}$ Provided that $t_{0}(x)$ has sufficiently many terms, depending on the nature of $B t$.
} 
to $\Gamma\left(w_{1}\right)$ terms. Hence $y_{0}(x)$ is independent of $r\left(=w_{1}\right)$ and the asymptotic relation is in the ordinary sense.

Consider the case $\mathrm{C}$. When $x$ is in $R$, for instance, in (18 a) $z$ is let to approach infinity along $B_{l}$. Again $c_{0,1}=0$ and the situation is as in the preceding case inasmuch as $x$ is restricted to $R^{l}$. Consider a solution $y_{l}(x)$ which, for $x$ in $R^{l}$, is asymptotic to $s(x)$ in the ordinary sense. Let $y_{r}(x)$ be a solution which has the same asymptotic form in $R^{r}$. We have

$$
y_{l}(x)=y_{r}(x)+c .
$$

Thus

$$
y_{l}(x)-c \sim s(x) \quad\left(x \text { in } R^{r}\right)
$$

An analogous fact holds true for $y_{r}(x)$ when $x$ is in $R^{l}$.

In the case $\mathrm{D}$ we obtain an asymptotic relationship valid in $R$ in the ordinary sense. To demonstrate this fact we need only to let $z$, in (8 a), approach infinity along $B_{l}$ or $B_{r}$. With $t_{0}$ suitably chosen, $c_{0,1}$ will then be seen to be zero.

In the remaining case when $\Re Q(x) \equiv 0$ the limit in (18 a) is taken as $z$ approaches infinity within $R$. Here again $c_{0,1}=0$ and $y_{0}(x)$ is independent of $w\left(=w_{1}\right)$. The solution $y_{0}(x)$ will be asymptotic to $s(x)$ in the ordinary sense, for $x$ in $R$.

The following Lemma can now be stated.

Lemma 3. Let $R$ be a region bounded by regular curves $B_{1}$ and $B_{r}$ (as specified above); that is, in $R$,

$$
\Re Q(x) \geqq 0 \quad\left(Q(x) \text { a polynomial in } x^{\frac{1}{k}}\right.
$$

Consider the equation

$$
y^{(1)}(x)=e^{Q(x)} x^{r} h(x)
$$

where $h(x)$ is analytic in $R(|x| \neq \infty)$ and

$$
h(x) \sim x(x)
$$

(a $\sigma$-series)

for $x$ in $R$. Let

$$
s(x)=e^{Q(x)} x^{r+\alpha} \eta(x)
$$

be the corresponding formal solution.

26-33617. Acta mathematica. 62. Imprimè le 7 novembre 1933. 
Case I. (2 I a) holds to $w$ terms ( $w$ sufficiently large). Then there exists a solution of (2I), $y(x)$, analytic in $R(|x| \neq \infty)$ and such that

$$
y(x) \sim s(x)
$$

to $\quad \Gamma(w)(<w)$ terms $[\lim (w)=\infty$, whenever $w$ can be made to approach infinity].

Here (22) holds in $R$ except that, whenever $\mathfrak{R} Q(x) \neq$ o while every region $\bar{R}(Q)$ (cf. $\$ 2$ ) contains neither $B_{l}$ nor $B_{r}\left(B_{l}\right.$ and $B_{r}$ with different limiting directions), there exists a solution such that (22) holds in $R^{l}$ [or $R^{r}$; cf. italics of Case C]: moreover, there exists a constant $c$ such that

(22 a)

$$
y(x) \cdots-c \sim s(x)
$$

for $x$ in $R^{r}\left[\right.$ or $\left.R^{l}\right]$.

Case II. (2 I a) holds in the ordinary sense. The results of Case I will then hold with the asymptotic relations (22), (22 a) valid in the ordinary sense.

Note. Suppose that interior $R$ we kave $\mathfrak{R} Q(x)<0$. This can be treated as the Case $\mathbf{E}$ had been treated above. The result would be precicely analogous to that obtained in Case $\mathrm{E}$.

6. Iterations. Let $R$ be a region, of the type of the region so denoted in $\$ 5$, bounded by curves $B_{l}$ and $B_{r}$. Consider a system of order $n$

$$
\begin{gathered}
Y^{(\mathbf{1})}(x)=Y(x) A(x) \\
A(x)=\left(a_{i, j}(x)\right), \quad Y(x)=\left(y_{i, j}(x)\right) \quad(i, j=1, \ldots n)
\end{gathered}
$$

where the $a_{i, j}(x)$ are analytic in $R(|x| \neq \infty)$ and

$$
\begin{gathered}
a_{i, j}(x) \sim \alpha_{i, j}(x)=a_{M}^{i, j} x^{\frac{M}{p}}+\cdots+a_{1}^{i, j} x^{1}+a_{0}^{i, j}+a_{-1}^{i, j} x^{-\frac{j}{p}}+\cdots \\
(i, j=\mathrm{I}, 2, \ldots n ; x \text { in } R)
\end{gathered}
$$

to $w$ ( $w$ suitably great) terms; that is,

$$
\text { (I b) } \begin{aligned}
a_{i, j}(x)=a_{M}^{i, j} x^{\frac{M}{p}}+\cdots+ & a_{0}^{i, j}+a_{-1}^{i, j} x^{-\frac{1}{p}}+\cdots+a_{-(v-1)}^{i, j} x^{-\left(\frac{w-1}{p}\right)} \\
& +\alpha_{u}^{i, j}(x) x^{-\frac{u}{p}}\left(\left|\alpha_{v}^{i, j}(x)\right| \leqq a_{v}^{i, j} ; \quad i, j=\mathrm{I}, \ldots n ; x \text { in } R\right) .
\end{aligned}
$$


Let

$$
S(x)=\left(s_{i, j}(x)\right)=\left(e^{Q_{i}(x)} x^{\left.r_{i, j} \sigma_{i, j}(x)\right)}\right.
$$

(cf. the statement in connection with $(7)$ in $\$$ I) be the corresponding matrix solution. It will be assumed that, interior $R$,

$$
\mathfrak{h} Q_{1}(x)=\mathfrak{R} Q_{2}(x)=\cdots=\mathfrak{R} Q_{\Gamma}(x)<\mathfrak{R} Q_{\Gamma+1}(x) \leqq \cdots \leqq \Re Q_{n}(x) .
$$

With a suitable $t$, let the matrix

$$
T(x)=\left(t_{i, j}(x)\right)=\left(e^{Q_{i}(x)} x^{\left.r_{i, j} \Gamma_{i, j}(x)\right)}\right.
$$

consist of elements obtained by omission, in the power series factors of the corresponding elements of $S(x)$, of the powers of $x$,

$$
x^{\frac{t+v}{k}} \quad(v=0, \mathrm{I}, 2, \ldots)
$$

Here $k$ is the lowest common multiple of $k_{i} \quad(i=\mathrm{I}, 2, \ldots n)$.

By means of the transformation

$$
Y(x)=Z(x) T(x), \quad Z(x)=\left(z_{i, j}(x)\right)
$$

the system (I) will go into the matrix equation

$$
Z^{(1)}(x)=Z(x) \Lambda(x)
$$

$$
A(x)=\left(\lambda_{i, j}(x)\right)=\left(T(x) A(x)-T^{(1)}(x)\right) T^{-1}(x) .
$$

Now

$$
T^{-1}(x)=\left(\bar{t}_{i, j}(x)\right)=\left(e^{-Q_{j}(x)} x^{r_{i, j}^{\prime}} \Gamma_{i, j}(x)\right),
$$

where $\Gamma_{i, j}(x) \quad(i, j=\mathrm{I}, 2, \ldots n)$ is in the form of a terminated $\sigma$-series. On writing

$$
T^{(1)}(x)=T(x) B(x),
$$$$
B(x)=\left(b_{i, j}(x)\right)
$$

it follows that

$$
b_{i, j}(x) \sim c_{i, j}(x) \quad(i, j=\mathrm{I}, 2, \ldots n ; x \text { in } R)
$$

to a number of terms so that

$$
\begin{gathered}
a_{i, j}(x)-b_{i, j}(x)=x^{-\frac{\sigma^{\prime}}{p}} c_{i, j}^{\sigma}(x) \\
\left(\left|c_{i, j}^{\sigma^{\prime}}(x)\right| \leqq c_{i, j}^{\sigma^{\prime}} ; i, j=\mathrm{I}, \ldots n ; x \text { in } R\right),
\end{gathered}
$$


where $\sigma^{\prime}=\sigma^{\prime}(w) \rightarrow \infty$, as $w \rightarrow \infty$ (whenever it is possible to let $\left.w \rightarrow \infty\right)$. In virtue of $(6 \mathrm{c})$ and $(6 \mathrm{~d})$ from $(6 \mathrm{a})$ it follows that

$$
I(x)=\left(e^{Q_{i, j}(x)} h_{i, j}(x) x^{-\frac{j}{p}}\right), \quad Q_{i, j}(x)=Q_{i}(x)-Q_{j}(x)
$$

$$
\text { i } h_{i, j}(x)|=| h_{i, j}^{\sigma}(x) \mid \leqq h_{i, j}^{\sigma}<h(\sigma) ;
$$

$$
i, j=\mathbf{1}, 2, \ldots n ; x \text { in } R] \text {, }
$$

where $\sigma=\sigma(w)$ and

$$
\lim _{w} \sigma(w)=\infty
$$

whenever $w$ can be indefinitely increased.

On taking note of $(7)$, the system (6) will be now solved in part by means of Product-integrals. ${ }^{1}$ In wiew of Lemma 1 ( $\left.\$ 3\right) ~ R$ consists of a finite number of proper regions (Definition of $\S 3$ ). Corresponding to any fixed $x$, in $R$, there will be associated a curve $C_{x}$, as specified in the Definition of $\S 3$ and satisfying the conditions of this Definition. Let $x_{0}\left(\left|x_{0}\right|>|x|\right)$ be some point on $C_{x}$. We form the matrix product

$$
\begin{aligned}
& Z_{(m)}=\left(z_{m: i, j}\right) \\
& =\left[I+\left(x_{1}-x_{0}\right) \Lambda\left(\Im_{1}\right)\right]\left[I+\left(x_{2}-x_{1}\right) \Lambda\left(\Im_{2}\right)\right] \cdots\left[I+\left(x_{m}-x_{m-1}\right) \Lambda\left(\Im_{m}\right)\right],
\end{aligned}
$$

where the points $x_{0}, x_{1}, x_{2}, \ldots x_{m-1}, x_{m}=\Im_{m}=x$ are consecutively ordered, as stated, along $C_{x}$ from $x_{0}$ to $x$. Moreover, as $m \rightarrow \infty$, the maximum $\left|x_{v}-x_{v-1}\right|$ $(\nu=\mathrm{I}, \ldots m)$ approaches zero, and $\mathfrak{J}_{\nu}(\nu=\mathrm{I}, \ldots m-\mathrm{I})$ is any point on the subarc of $C_{x}$ whose end points are $x_{v-1}, x_{v}$. According to the known theory of Productintegrals, the limiting matrix

$$
Z_{0}(x)=\left(x_{0: i, j}(x)\right)=\lim _{m=\infty} Z_{m}=\int_{x_{i}}^{x}(A(x) d x+I)
$$

will represent a matrix solution of (6). The elements in each row will constitute a solution. Examine now in greater detail the matrix $Z_{(m)}$. On letting $x_{k}-x_{k-1}=v_{k}$ we have

\footnotetext{
${ }^{1}$ Cf., for instance, L. Schlesinger, Vorlesungen über lineare Differentialgleichungen, I 908.
} 
(8 b) $\quad Z_{(m)}=I+\sum_{k_{1}=1}^{m} v_{k_{1}} \Lambda\left(\Im_{k_{1}}\right)+\sum_{k_{1}<k_{2}}^{m} v_{k_{1}} v_{k_{2}} \Lambda\left(\Im_{k_{1}}\right) \Lambda\left(\Im_{k_{2}}\right)+$

$$
\begin{aligned}
& +\sum_{k_{1}<\ldots<k_{s}}^{m} v_{k_{1}} v_{k_{2}} \ldots v_{k_{3}} \Lambda\left(\Im_{k_{1}}\right) \Lambda\left(\Im_{k_{2}}\right) \ldots \Lambda\left(\widetilde{\Im}_{k_{3}}\right)- \\
& \quad+v_{1} v_{2} \ldots v_{m} \Lambda\left(\Im_{1}\right) \Lambda\left(\Im_{2}\right) \ldots \Lambda\left(\Im_{m}\right) \\
& =I+\sum_{s=1}^{m} L_{s} ; \quad L_{s}=\left(l_{8: i, j}\right) ; \quad I=\left(\delta_{i, j}\right) .
\end{aligned}
$$

Substitution of $(7)$ will result in the following expression for $l_{s: i, j}(i, j=\mathbf{1}, \ldots n)$

(9) $l_{s: i, j}=\sum_{k_{1}<\ldots<k_{g}}^{m}\left\{\left(v_{k_{1}} \Im_{k_{1}}^{-\frac{\sigma}{p}}\right)\left(v_{k_{2}} \Im_{k_{2}}^{-\frac{\sigma}{k}}\right) \ldots\left(v_{k_{s}} \widetilde{\jmath}_{k_{s}} \frac{\sigma}{p}\right)\right.$

In (9)

$$
\left.\cdot \sum_{\lambda_{1}, \ldots \lambda_{s-1}=1}^{n} e^{W_{\lambda_{1}, \ldots, j \lambda_{s-1}}^{k_{1}, \ldots h_{i, \lambda_{1}}}\left(\widetilde{\Im}_{k_{1}}\right) h_{\lambda_{1}, \lambda_{2}}\left(\mathfrak{\Im}_{k_{2}}\right) \ldots h_{\lambda_{s-1}, j}\left(\widetilde{\Im}_{k_{s}}\right)}\right\}
$$

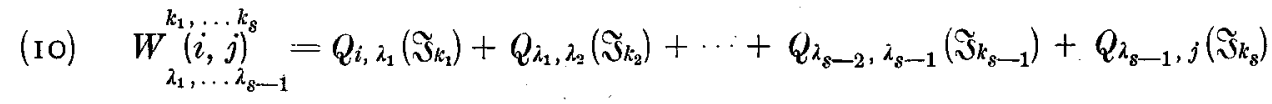

$$
\begin{aligned}
=Q_{i, j}(x)+\left[Q_{j, i}(x)-Q_{j, i}\left(\Im_{k_{g}}\right)\right]+\left[Q_{\lambda_{s-1}, i}\left(\Im_{k_{g}}\right)-Q_{\lambda_{x-1}, i}\left(\Im_{k_{s-1}}\right)\right] \\
+\cdots+\left[Q_{\lambda_{2}, i}\left(\Im_{k_{3}}\right)-Q_{\lambda_{2}, i}\left(\Im_{k_{2}}\right)\right]+\left[Q_{\lambda_{1}, i}\left(\Im_{k_{2}}\right)-Q_{\lambda_{1}, i}\left(\Im_{k_{1}}\right)\right]{ }^{1}
\end{aligned}
$$

Application of Lemma I $\left(\S_{3}\right)$ enables us to assert, in view of the satisfied conditions of the Definition $(\S 3)$ and in view of ( $(\dot{0})$ ), that there exists a constant $g(=\log w)$, independent of $x, x_{0}$ and $C_{x}$, such that we have

$$
\begin{aligned}
& \Re \underset{\lambda_{1}, \ldots \lambda_{8-1}}{\stackrel{k_{1}, \ldots k_{s}}{(i, j)}} \leqq \Re Q_{i, j}(x)+\log w \\
& (i=\mathrm{I}, 2, \ldots \Gamma ; j=\mathrm{I}, 2, \ldots n)
\end{aligned}
$$

for all $x$ in $R$.

Thus, on using ( $8 \mathrm{~b}),(9)$ and ( $\mathrm{I})$, it is observed that the elements in the first $\Gamma$ rows of $Z_{(m)}$ satisfy the inequalities

\footnotetext{
${ }^{1}$ Here $Q_{\sigma, s}=Q_{\sigma}-Q_{s}$.
} 
( 12 )

$$
\begin{aligned}
& \left|z_{m: i, j}-\delta_{i, j}\right|=\left|\sum_{s=1}^{m} l_{8: i, j}\right| \\
& \leqq w\left|e^{Q_{i, j(x)}}\right| \sum_{s=1}^{m} \sum_{k_{1}<\cdots<k_{s}=1}^{m}\left\{\left|\left(v_{k_{1}} \mathfrak{\Im}_{k_{1}}-\frac{\sigma}{p}\right)\left(v_{k_{2}} \mathfrak{\Im}_{k_{g}}-\frac{\sigma}{p}\right) \ldots\left(v_{k_{g}} \mathfrak{\Im}_{k_{\varepsilon}}-\frac{\sigma}{p}\right)\right| \cdot\right. \\
& \left.\sum_{\lambda_{1}, \ldots \lambda_{s-1}=1}^{n}\left|h_{i, \lambda_{1}}\left(\widetilde{J}_{k_{1}}\right) h_{\lambda_{1}, \lambda_{3}}\left(\Im_{\mathfrak{J}_{2}}\right) \ldots h_{\lambda_{s-1}, j}\left(\Im_{\mathfrak{J}_{g}}\right)\right|\right\}
\end{aligned}
$$

and, by (7 a), we have with $h=n h(\sigma)$

( 12 a)

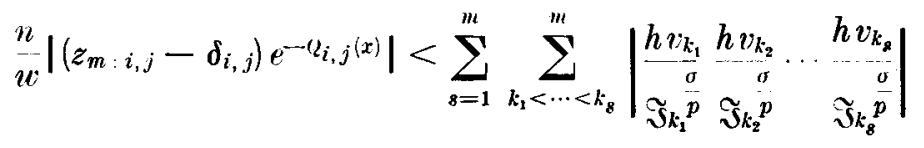

$$
\begin{aligned}
& =-\mathrm{I}+\prod_{\nu=1}^{m}\left(\mathrm{I}+h\left|v_{\nu}\right| \Im_{\nu}-\frac{\sigma}{p}\right)<e^{h \sum_{v=1}^{m}\left|v_{\nu}\right|\left|\Im_{v}\right|-\frac{\sigma}{p}}-\mathrm{I} \\
& (i=\mathrm{I}, \ldots \Gamma ; j=\mathrm{I}, 2, \ldots n) .
\end{aligned}
$$

Now, with $\frac{\sigma}{p}$ sufficiently great,

$$
\lim _{m} \sum_{\nu=1}^{m}\left|v_{v}\right|\left|\Im_{\nu}\right|^{-\frac{\sigma}{p}}=\int_{z=x_{0}}^{z=x}\left|\frac{d z}{\frac{\sigma}{z^{p}}}\right|<\int_{c_{x}}\left|\frac{d z}{\frac{\sigma}{z^{p}}}\right|<\frac{c}{\left|\frac{\sigma}{x^{\frac{\sigma}{p}}-\lambda}\right|}
$$

where $\lambda$ and $c$ are independent of $x, x_{0}$ and $\sigma$. This inequality is a consequence of Lemma I $(\S 3)$; by virtue of this Lemma condition $3^{\circ}$, of the Definition of $\S 3$, is satisfied. Accordingly, from (I 2 a) and ( 3 ) it follows that

$$
\begin{gathered}
\frac{n}{w}\left|\left(z_{0: i, j}(x)-\delta_{i, j}\right) e^{-Q_{i, j}(x)}\right|<e^{\left|\frac{h c}{x_{x}^{\frac{o}{p}-i}}\right|}-\mathrm{I} \\
(i=\mathrm{I}, \ldots \Gamma ; j=\mathrm{I}, 2, \ldots n) .
\end{gathered}
$$

The last member above is independent of $x_{0}$. Let $x_{0}$ recede to infinity along $C_{x}$. The limits

$$
z_{i, j}(x)=\lim _{x_{0}=\infty} z_{0: i, j}(x) \quad(i=\mathrm{I}, \ldots \Gamma ; j=\mathrm{I}, 2, \ldots n)
$$

will exist and 


$$
\begin{gathered}
\left|\left(z_{i, j}(x)-\delta_{i, j}\right) e^{-Q_{i, j}(x)}\right|<\frac{d(\sigma)}{\left|\frac{\sigma}{x^{p}-\lambda}\right|} \\
(i=\mathrm{I}, 2, \ldots \Gamma ; j=\mathrm{I}, 2, \ldots n)
\end{gathered}
$$

where $d(o)$ is independent of $x$ and $C_{x}$. Thus the Product-integral (8 a) can be extended to infinity in the first $I$ rows. The limiting elements (I4 a) will constitute $\Gamma$ solutions of (6). It is clear that Product-integration will constitute a convergent process, for the elements of the first $\Gamma$ rows, when the path is extended along any regular curve situated in $R$. The choice of $C_{x}$ was merely made in order to secure the inequalities (15). In view of the above and of the properties of these integrals the functions (14 a) will be analytic in $R(|x| \neq \infty)$; they will be constituent elements of $\Gamma$ solutions, in $R$, of $(6){ }^{1} \quad$ Moreover, they will satisfy (15) for $x$ in $R$.

Returning to the system (I), $\Gamma$ solutions will be given with the aid of (5) and the $\Gamma$ solutions, just obtained, of the system (6). Now

$$
\begin{gathered}
z_{i, j}(x)=\delta_{i, j}+e^{Q_{i, j}(x)}{ }_{o} z_{i, j}(x) x^{-\left(\frac{\sigma}{p}-\lambda\right)} \\
{\left[\left.\right|_{a} z_{i, j}(x) \mid<d(\sigma) ; \quad(i=\mathrm{I}, \ldots \Gamma ; j=\mathrm{I}, 2, \ldots n) ; \quad x \text { in } R\right] .}
\end{gathered}
$$

Thus

$$
\begin{aligned}
& y_{i, j}(x)=\sum_{v=1}^{n} z_{i, v}(x) t_{\boldsymbol{v}, j}(x) \\
& =\sum_{v=1}^{n}\left[\delta_{i, v}+e^{Q_{i, v}(x)}{ }_{\sigma} z_{i, v}(x) x^{-\left(\frac{\sigma}{p}-\lambda\right)}\right] e^{Q_{\nu}(x)} x^{r_{v}, j} \Gamma_{\nu, j}(x) \\
& =e^{Q_{i}(x)}\left[x^{r_{i, j}} \Gamma_{i, j}(x)+x^{-\left(\frac{\sigma}{p}-\lambda\right)} \sum_{\nu=1}^{n} x^{r_{\nu, j}} z_{i, v}(x) \Gamma_{\nu, j}(x)\right] .
\end{aligned}
$$

Accordingly, the $y_{i, j}(x)(i=\mathrm{I}, 2, \ldots \Gamma ; j=\mathrm{I}, \ldots n)$ are seen to be asymptotic, in $R$, to the $s_{i, j}(x)$, respectively. The asymptotic relations here will be valid to $\eta(w)$ terms, where $\lim \eta(w)=\infty$, whenever $w$ (of ( $\mathrm{I} \mathbf{b})$ ) can be made arbitrarily great.

Lemma 4. Consider the system specified by (I), (I a), (I b), (2). Let $R$ be a region, bounded by two regular curves extending to infinity, in which an ordering (3) is maintained. Let $T(x)$ be defined by (4), with $t$ suitably great. Let $A(x)$ be

${ }^{1}$ They will be independent of the path of Product-integration, inasmuch as the path extends to infinity and convergence conditions are satisfied. 
defined by (6a). Consider the functions $z_{0: i, j}(x)(i=1, \ldots \Gamma ; j=1,2, \ldots n)$ which are defined, for $x$ in $R$, with the aid of the Product-integral $(8 \mathbf{a}) ; x_{0}$ being in $R$. The limits

$$
\begin{gathered}
z_{i, j}(x)=\lim _{x_{0}} z_{0: i, j}(x) \\
(i=1,2, \ldots \Gamma ; j=1,2, \ldots n ; \quad x \text { in } R)
\end{gathered}
$$

will exist, as $x_{0}$ approaches infinity along amy regular curve extending in $R$. The functions

$$
\begin{aligned}
& { }^{{ }^{w} y_{i, j}(x)=\sum_{i=1}^{n} z_{i, i}(x) t_{i, j}(x)} \\
& (i=\mathrm{I}, 2, \ldots \Gamma ; j=\mathrm{I}, \ldots n)
\end{aligned}
$$

will represent constituent elements of $\Gamma$ solutions of the system ( $\mathrm{I}$ ) and they will be analytic in $R(|x| \neq \infty)$. Moreover,

$$
\begin{gathered}
{ }_{w} y_{i, j}(x) \sim e^{Q_{i}(x)} x^{r_{i, j} \sigma_{i, j}(x)} \\
(i=\mathrm{I}, 2, \ldots I ; j=\mathrm{I}, 2, \ldots n)
\end{gathered}
$$

for $x$ in $R$. Here the asymptotic relations are valid to $\eta(w)$ terms, where $\eta(w)$ can be made arbitrarily great, whenever it is possible to increase $w(o f(\mathrm{I} b))$ indefinitely.

In the course of proving the Fundamental Existence Theorem $(\$ 7)$ it will be established that, whenever $w$ (in ( $\mathrm{I}$ b)) can be made to approach infinity, the functions $(17 \mathrm{a})$ are independent of $w$ so that $(\mathrm{I} / \mathrm{b})$ holds in the ordinary sense.

7. The Fundamental Existence Theorem. The developments of the preceding sections enable us to undertake the proof of the main theorem of this paper.

The Fundamental Existence Theorem. Consider a singular equation $\left(c f . \S \mathrm{I}^{\circ}\right)$, of order $n$,

$$
L_{n}(y)=\text { o }
$$

Let $R_{i}(i=\mathrm{I}, 2, \ldots N)$ be the corresponding regions $(\mathrm{1} 6 \mathrm{a} ; \S 2)$. Consider a particular region $R_{i}$. If there exist ro functions $(18 ; \$ 2)$ such that $(18 \mathrm{a} ; \$ 2)$ holds, or if the boundaries of $R_{i}$ have the same limiting directions, there exists a full set of solutions $y_{v}(x)(\nu=\mathrm{I}, 2, \ldots n)$, of $(\mathbf{A})$, such that

$$
\begin{gathered}
y_{v}(x) \sim e^{Q_{\nu}(x)} x^{r_{v}} \sigma_{\nu}(x)=s_{v}(x) \\
\left(\nu=\mathrm{I}, 2, \ldots n ; \quad x \text { in } R_{i}\right) .
\end{gathered}
$$


If there exist functions $(\mathrm{I} 8 ; \S 2)$ such that $(\mathrm{I} 8 \mathrm{a} ; \S 2)$ holds, while the boundaries of $R_{i}$ have different limiting directions, consider the two subregions $R_{i}^{\prime}$ and $R_{i}^{\prime \prime}$ of $R_{i}$ as specified in the italicized statement following (I8 a; $\$ 2$ ). In this case, also, there will exist a full set of solutions, satisfying (i), for $x$ in $R_{i}^{\prime}$, and another set of solutions, satisfying (I) in $R_{i}^{\prime \prime}$. In (I) the asymptotic relations are in the ordinary sense and the $s_{i}(x)$ are formal solutions.

In virtue of the connection between single equations of order $n$ and systems, an analogous theorem will hold for singular systems $\left(B ; \S I^{\circ}\right)$.

Proof. Let $R$ denote any particular region for which the asymptotic relations ( $\mathrm{I}$ ) had been asserted in the formulation of the above theorem. Interior $R$ the following ordering will be maintained

$$
\begin{gathered}
(2) \quad \Re Q_{1}(x)=\Re Q_{2}(x)=\cdots=\Re Q_{\Gamma_{1}}(x)<\Re Q_{\Gamma_{1}+1}(x)=\Re Q_{\Gamma_{1}+2}(x)=\cdots=\Re Q_{\Gamma_{2}}(x) \\
<\Re Q_{\Gamma_{2}+1}(x)=\cdots=\Re Q_{\Gamma_{3}}(x)<\cdots<\Re Q_{\Gamma_{\gamma-1}+1}(x)=\Re Q_{\Gamma_{\gamma-1}+2}(x)=\cdots=\Re Q_{\Gamma_{\gamma}}(x) \\
\left(\mathrm{I} \leqq \Gamma_{1}<\Gamma_{2}<\cdots<\Gamma_{\gamma-1}<I_{\gamma}=n\right) .
\end{gathered}
$$

Apply now Lemma $4(\S 6)$ to the system (D; § I),

$$
Y^{(1)}(x)=Y(x) D(x)
$$

which corresponds to the equation $L_{n}(y)=0$. It is concluded that there exist $\Gamma_{1}$ solutions,

$$
{ }_{1} y_{i}(x)={ }_{1} y_{i, 1}(x)
$$$$
\left(i=1,2, \ldots T_{1}\right),
$$

analytic in $R(|x| \neq \infty)$ and such that

$$
\begin{gathered}
{ }_{1} y_{i}(x) \sim e^{Q_{i}(x)} x^{r_{i}} \sigma_{i}(x) \\
\left(i=\mathrm{1}, 2, \ldots \Gamma_{1} ; x \text { in } R\right)
\end{gathered}
$$

to $\eta_{1}$ terms. These functions will correspond to a suitable choice of the matrix $T(x)$, which is used in the Iteration of Lemma 4. By the same Lemma, the functions

$$
{ }_{1} y_{i, j}(x)={ }_{1} y_{i}^{(j-1)}(x) \quad\left(i=\mathrm{I}, 2, \ldots r_{1} ; j=\mathbf{I}, 2, \ldots n\right)
$$

will be asymptotic, in $R$, to the formal series

$$
s_{i, j}(x)=s_{i}^{(j-1)}(x) \quad \text { (to } \eta_{1} \text { terms), }
$$

respectively.

27-33617. Acta mathematica. 62. Imprimé le 8 novembre 1933. 
The equation

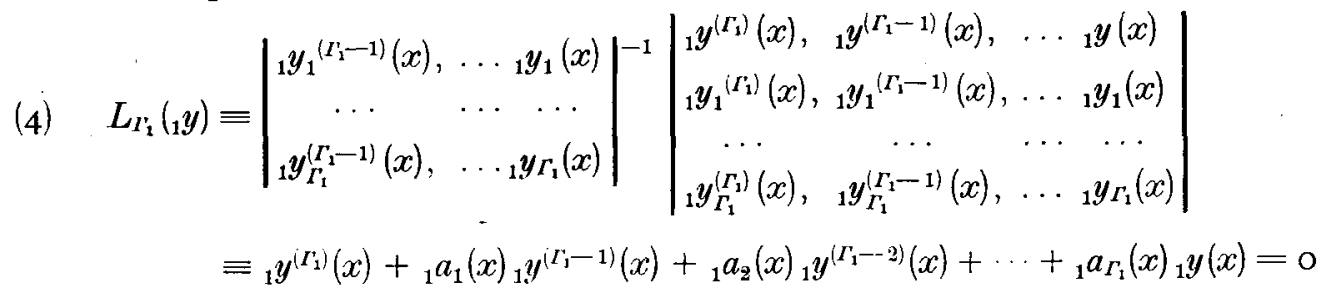

will be satisfied by ${ }_{1} y_{i}(x) \quad\left(i=\mathrm{I}, 2, \ldots \Gamma_{1}\right)$. The coefficients ${ }_{1} a_{i}(x) \quad\left(i=\mathrm{I}, 2, \ldots \Gamma_{1}\right)$ will be analytic in $K(|x| \neq \infty)$. The formal equation

(4 a) $\quad L_{\Gamma_{1}}^{*}\left({ }_{1} y\right) \equiv{ }_{1} y^{\left(\Gamma_{1}\right)}(x)+{ }_{1} \alpha_{1}(x){ }_{1} y^{\left(\Gamma_{1}-1\right)}(x)+{ }_{1} \alpha_{2}(x){ }_{1} y^{\left(\Gamma_{1}-2\right)}(x)+\cdots+{ }_{1} \alpha_{\Gamma_{1}}(x){ }_{1} y(x)=0$

corresponding to $(4)$, is obtained by replacing the functions

$$
{ }_{1} y_{i}^{(j)}(x) \quad\left(i=\mathrm{I}, 2, \ldots I_{1} ; j=0,1, \ldots I_{1}\right)
$$

occurring in (4), by the formal series $\varepsilon_{i}^{(j)}(x)$, respectively. In view of the remarks made in $\$ 1$, the coefficients in $(4 \mathrm{a})$ are seen to be formal series of the type of $\left(\mathbf{I} ; \S \mathbf{I}^{\circ}\right)$. Equation $(4 \mathrm{a})$ possesses $\Gamma_{1}$ linearly independent formal solutions

$$
s_{i}(x)={ }_{1} s_{i}(x) \quad\left(i=\mathrm{I}, 2, \ldots \Gamma_{1}\right)
$$

In virtue of the asymptotic relations $(3 \mathrm{~b})$ satisfied by the functions $(3 \mathrm{c})$ it follows that

$$
{ }_{1} a_{i}(x) \sim{ }_{1} \alpha_{i}(x) \quad\left(i=1,2, \ldots \Gamma_{1} ; x \text { in } R\right)
$$

to $\alpha_{1}$ terms. By taking $\eta_{1}$ sufficiently great (which can be accomplished by suitable choice of $T(x)) \alpha_{1}$ can be made arbitrarily great. The equation (4) has in common with $L_{n}(y)=o$ a set of $I_{1}^{\prime}$ linearly independent solutions

$$
{ }_{1} y_{i}(x)=y_{i}(x) \quad\left(i=1,2, \ldots \Gamma_{1}\right) .
$$

Every solution of (4), beíng expressible as a linear combination with constant coefficients of the functions (6), will necessarily satisfy $L_{n}(y)=0$. Hence there is an analytic factorization

$$
L_{n}\left({ }_{1} y\right) \equiv L_{n-\Gamma_{\mathfrak{1}}} L_{\Gamma_{1}}(y)
$$

$$
L_{n-\Gamma_{1}}\left({ }_{1} z\right) \equiv{ }_{1} z^{\left(n-\Gamma_{1}\right)}(x)+{ }_{1} b_{1}(x){ }_{1} z^{\left(n-\Gamma_{1}+1\right)}(x)+\cdots+{ }_{1} b_{n-\Gamma_{1}}(x){ }_{1} z(x)
$$

where the ${ }_{1} b_{i}(x)\left(i=\mathrm{I}, 2, \ldots n-\Gamma_{1}\right)$ are analytic in $R(|x| \neq \infty)$. Similarly, there is a corresponding formal factorization 


$$
\left.\left.L_{n}^{*}{ }_{1} y\right) \equiv L_{n-I_{1}}^{*} L_{I_{1}}^{*}{ }_{1} y\right),
$$

$$
L_{n-\Gamma_{1}}\left({ }_{1} z\right) \equiv{ }_{1} z^{\left(n-\Gamma_{1}\right)}(x)+{ }_{1} \beta_{1}(x){ }_{1} z^{\left(n-\Gamma_{1}-1\right)}(x)+\cdots+{ }_{1} \beta_{n-\Gamma_{1}}(x){ }_{1} z(x),
$$

where the formal series ${ }_{1} \beta_{i}(x)$ are of the type $\left(\mathrm{I} ; \S \mathrm{I}^{\circ}\right)$ and $L_{n}^{*}\left({ }_{1} y\right)$ is a formal operator corresponding to $L_{n}(y)$. In connection with this formal reducibility (and similar ones to follow) it is essential to note that, if the formal series in $L_{n}^{*}\left({ }_{1} y\right)$ are in powers of $x^{\frac{1}{k}}\left(k\right.$, integer), the formal series ${ }_{1} \beta_{i}(x)$ in $L_{n-\Gamma_{1}}^{*}\left({ }_{1} z\right)$ will be in powers of $x^{\frac{1}{p}}$, where $p$ may be an integer different ${ }^{1}$ from $k$. The equation

$$
L_{n-\Gamma_{1}}^{*}\left({ }_{1} z\right)=\mathrm{o}
$$

will be satisfied by $\left(n-\Gamma_{1}\right)$ linearly independent formal solutions

$$
\begin{aligned}
& L_{\Gamma_{1}}^{*}\left(s_{\Gamma_{1}+i}(x)\right)={ }_{1} f_{\Gamma_{1}+i}(x)=e^{Q_{\Gamma_{1}+i}(x)} x^{1} f_{\Gamma_{1}+i} \varphi_{\Gamma_{1}+i}(x) \\
& \left(i=\mathrm{I}, 2, \ldots n-\Gamma_{1}\right),
\end{aligned}
$$

where the ${ }_{1} \varphi_{T_{1}+i}(x)$ are $\sigma$-series (cf. $\left.\S \mathrm{I}\right)$. This is a consequence of $(7 \mathrm{~b}$ ) and of the fact that, formally, $L_{n}^{*}\left(s_{\Gamma_{1}+i}(x)\right)=0$. It is clear that

$$
{ }_{1} b_{i}(x) \sim{ }_{1} \beta_{i}(x) \quad\left(i=\mathrm{I}, 2, \ldots n-\Gamma_{1} ; x \text { in } R\right)
$$

to $\beta_{1}$ terms. Here $\beta_{1}$ can be made arbitrarily great by a suitable choice of $T(x)$.

There exists a systen of order $n-\Gamma_{1}$, of type $(\mathrm{D} ; \S \mathrm{I})$, which corresponds to the equation $L_{n \rightarrow \Gamma_{1}}\left({ }_{1} z\right)=0$. Application of Lemma 4 to this system is possible. Accordingly, it can be asserted that there exist $\Gamma_{2}-\Gamma_{1}$ solutions of the equation $L_{n-\Gamma_{1}}\left({ }_{1} z\right)=0$, analytic in $R(|x| \neq \infty)$, such that

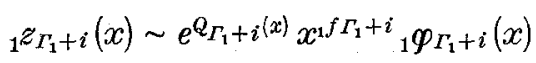

$$
\begin{aligned}
& \left(i=\mathrm{I}, 2, \ldots \Gamma_{2}-\Gamma_{1} ; x \text { in } R\right)
\end{aligned}
$$

to $z_{1}$ terms. By originally defining $T(x)$ as $S(x)$ with a suitably great number of terms retained, $z_{1}$ can be made arbitrarily great.

In addition to the $\Gamma_{1}$ solutions (6) the equation $L_{n}(y)=0$ is seen to possess $\Gamma_{2}-\Gamma_{1}$ solutions

$$
\text { (I I) }{ }_{2} y_{\Gamma_{1}+i}(x) \quad\left(i=\mathrm{I}, 2, \ldots \Gamma_{2}-\Gamma_{1}\right) \text {, }
$$

where any particular solution ${ }_{2} y_{\Gamma_{1}+i}(x)$ satisfies the non-homogeneous equation

$$
\text { (I I a) } \quad L_{\Gamma_{1}}\left({ }_{2} y\right)={ }_{1} z_{\Gamma_{1}+i}(x) \quad\left(\mathrm{I} \leqq i \leqq \Gamma_{2}-\Gamma_{1}\right) \text {. }
$$

${ }^{1}$ In certain cases, as for instance when the $n$ formal solutions of $L_{n}(y)=0$ are given by $n$ determinations of the same series, $p$ will certainly be different from $k$. 
Write

$$
\begin{gathered}
\left({ }_{1} y_{i}{ }^{(j-1)}(x)\right)^{-1}=\left({ }_{1} \bar{y}_{i, j}(x)\right) \\
\left(i, j=\mathrm{I}, 2, \ldots \Gamma_{1}\right) .
\end{gathered}
$$

Then, by $(4 ; \$ I)$, a solution of (I I a) can be given by the formula

$$
{ }_{2} y \Gamma_{1}+i(x)=\sum_{\lambda=1}^{\Gamma_{1}}{ }_{1} y_{\lambda}(x) \int{ }_{1} z_{\Gamma_{1}+i}(x)_{1} \bar{y}_{\Gamma_{i}}, \lambda(x) d x \text {. }
$$

In view of the conditions imposed on a region $R$ (cf. the formulation of the Theorem) the integration methods of Lemma $3(\S 5)$ are applicable. Thus, ${ }_{2} y_{\Gamma_{1}+i}(x)$, as defined by (12) and with the integrals extended according to Lemma 3 , is analytic in $R(|x| \neq \infty)$ and

$$
\begin{gathered}
{ }_{2} y_{\Gamma_{1}+i}(x) \sim{ }_{2} s_{\Gamma_{1}+i}(x)=e^{Q_{\Gamma_{1}}+i}{ }(x) x^{\mathrm{s}} r_{i}{ }_{2} \sigma_{\Gamma_{1}+i}(x) \\
{\left[x \text { in } R ; \quad{ }_{2} \sigma_{\Gamma_{1}+i} \text { a } \sigma \text {-series }\right]}
\end{gathered}
$$

to $\eta_{2}$ terms. Such a construction can be made and a relation (12 a) can be obtained for $i=\mathrm{I}, 2, \ldots \Gamma_{2}-I_{1}$.

We form now an equation of order $I_{2}^{\prime}$,

$$
L_{\Gamma_{2}}\left({ }_{2} y\right) \equiv{ }_{2} y^{\left(\Gamma_{2}\right)}(x)+{ }_{2} a_{1}(x){ }_{2} y^{\left(\Gamma_{2}-1\right)}(x)+\cdots+{ }_{2} a_{\Gamma_{2}}(x){ }_{2} y(x)=0 \text {, }
$$

which is satisfied by the $I_{2}^{*}$ linearly independent solutions

$$
\begin{array}{cr}
{ }_{2} y_{i}(x)={ }_{1} y_{i}(x) & \left(i=\mathrm{I}, 2, \ldots \Gamma_{1}\right), \\
{ }_{2} y_{\Gamma_{1}+i}(x) & \left(i=\mathrm{I}, 2, \ldots \Gamma_{2}-\Gamma_{1}\right) .
\end{array}
$$

This equation is formed, on the basis of the ${ }_{2} y_{i}(x)\left(i=1,2, \ldots \Gamma_{2}\right)$ in a way entirely analogous to that in which the equation (4) was constructed with the aid of the ${ }_{1} y_{i}(x)\left(i=\mathrm{I}, 2 \ldots \Gamma_{1}\right)$. The coefficients ${ }_{2} a_{i}(x)\left(i=\mathrm{I}, \ldots \Gamma_{2}\right)$ will be analytic in $R(|x| \neq \infty)$. To (13) there will correspond a formal equation

$$
L_{\Gamma_{2}}^{*}\left({ }_{2} y\right) \equiv{ }_{2} y^{\left(\Gamma_{2}\right)}(x)+{ }_{2} \alpha_{1}(x){ }_{2} y^{\left(\Gamma_{2}-1\right)}(x)+\cdots+{ }_{2} \alpha_{\Gamma_{2}}(x)_{2} y(x)=0 \text {. }
$$

It is satisfied by $\Gamma_{2}$ linearly independent formal solutions

$$
\begin{array}{cr}
{ }_{2} s_{i}(x)={ }_{1} s_{i}(x) & \left(i=\mathrm{I}, 2, \ldots \Gamma_{1}\right), \\
{ }_{2} s_{\Gamma_{1}+i}(x) & \left(i=\mathrm{I}, \ldots \Gamma_{2}-\Gamma_{1}\right) .
\end{array}
$$

The ${ }_{2} \alpha_{i}(x)$ will be of the type of $\left(\mathrm{I} ; \S_{\mathrm{I}^{\circ}}\right)$. Similar to the relations (5) we now have

$$
{ }_{2} a_{i}(x) \sim{ }_{2} \alpha_{i}(x) \quad\left(i=\mathrm{I}, 2, \ldots I_{2} ; x \text { in } R\right)
$$


to $\alpha_{2}$ terms, where $\alpha_{2}$ may be made as large as desired. ${ }^{1}$ In place of $(7)$ the following analytic factorization will hold

$$
L_{n}\left({ }_{2} y\right) \equiv L_{n-\Gamma_{2}} L_{\Gamma_{2}}\left({ }_{2} y\right),
$$

(16 a) $\quad L_{n-\Gamma_{2}}\left({ }_{2} z\right) \equiv{ }_{2} z^{\left(n-\Gamma_{2}\right)}(x)+{ }_{2} b_{1}(x){ }_{2} z^{\left(n-\Gamma_{2}-1\right)}(x)+\cdots+{ }_{2} b_{n-\Gamma_{3}}(x)_{2} z(x)$.

The ${ }_{2} b_{i}(x)$ will be analytic in $R(|x| \neq \infty)$. There will be a corresponding formal factorization

$$
\begin{gathered}
\left.L_{n}^{*}{ }_{2} y\right) \equiv L_{n-\Gamma_{2}}^{*} L_{\Gamma_{2}}^{*}\left({ }_{2} y\right) \\
L_{n-I_{2}}^{*}\left({ }_{2} z\right) \equiv{ }_{2} z^{\left(n-\Gamma_{2}\right)}(x)+{ }_{2} \beta_{1}(x){ }_{2} z^{\left(n-\Gamma_{2}-1\right)}(x)+\cdots+{ }_{2} \beta_{n-I_{2}}(x)_{2} z(x),
\end{gathered}
$$

and

$$
{ }_{2} b_{i}(x) \sim{ }_{2} \beta_{i}(x)
$$

$$
\left[x \text { in } R ;{ }_{2} \beta_{i}(x) \text { of type }\left(\mathrm{I} ; \S \mathrm{I}^{\circ}\right) ; i=\mathrm{I}, 2, \ldots n-\Gamma_{2}\right] \text {. }
$$

The formal equation $\left.L_{n-\Gamma_{2}}^{*}{ }_{2} z\right)=0$ will be satisfied by $n-\Gamma_{2}$ linearly independent series

$$
\begin{gathered}
L_{\Gamma_{2}}^{*}\left(s_{\Gamma_{2}+i}(x)\right)={ }_{2} f_{\Gamma_{2}+i}(x)=e^{Q_{\Gamma_{2}+i}(x)} x^{2} f_{\Gamma_{2}+i}{ }_{2} \varphi_{\Gamma_{2}+i}(x) \\
{\left[{ }_{1} \varphi_{\Gamma_{2}+i}(x) \text { a } \sigma \text {-series; } i=\mathrm{I}, 2, \ldots n-\Gamma_{2}\right] .}
\end{gathered}
$$

To the system of order $\left(n-\Gamma_{2}\right)$, corresponding to the equation $L_{n-\Gamma_{2}}\left({ }_{2} z\right)=0$, Lemma 4 can be applied. Thus, there exist $\Gamma_{3}-\Gamma_{2}$ solutions, analytic in $R(|x| \neq \infty)$ such that

$$
\begin{gathered}
{ }_{2} z_{\Gamma_{2}+i}(x) \sim e^{Q_{\Gamma_{2}}+i(x)} x^{\mathrm{a} f_{\Gamma_{2}}+i_{2}} \varphi_{I_{2}+i}(x) \\
{\left[i=\mathrm{I}, 2, \ldots \Gamma_{3}-\Gamma_{2} ; x \text { in } R\right]}
\end{gathered}
$$

to $z_{2}$ terms.

We solve now

$$
\left.L_{\Gamma_{2}}{ }_{3} y\right)={ }_{2} z_{\Gamma_{2}+i}(x) \quad\left(\mathrm{I} \leqq i \leqq \Gamma_{3}-\Gamma_{2}\right)
$$

obtaining $\Gamma_{\mathbf{3}}-\Gamma_{2}$ solutions of $L_{n}(y)=0$

where

$$
\begin{gathered}
{ }_{3} y_{\Gamma_{2}+i}(x)=\sum_{\lambda=1}^{\Gamma_{2}}{ }_{2} y_{\lambda}(x) \int{ }_{2} z_{\Gamma_{2}+i}(x)_{2} \bar{y}_{\Gamma_{2}, \lambda}(x) d x \\
\left(i=\mathrm{I}, 2, \ldots \Gamma_{3}-\Gamma_{2}\right)
\end{gathered}
$$

$$
\left.\left({ }_{2} y_{i}^{(j-1)}(x)\right)^{-1}={ }_{(2} \bar{y}_{i, j}(x)\right) \quad\left(i, j=\mathrm{I}, \ldots \Gamma_{2}\right) .
$$

${ }^{1}$ However, for the present, it cannot be asserted that the ${ }_{2} a_{i}(x)$ are independent of $\alpha_{2}$. 
In view of the known asymptotic forms of the involved elements application of Lemma 3 will result in the relations

$$
\begin{aligned}
& { }_{\mathrm{s}} y_{\Gamma_{2}+i}(x) \sim{ }_{3} s_{\Gamma_{z}+i}(x)=e^{Q_{\Gamma_{2}+i}(x)} x^{\mathrm{s}^{r_{i}}{ }_{\mathrm{g}} \sigma_{\Gamma_{\mathrm{g}}+i}(x)} \\
& \left.{ }_{3} \sigma_{\Gamma_{3}+i} \text { a } \sigma \text { series; } i=1,2, \ldots \Gamma_{3}-\Gamma_{2}\right),
\end{aligned}
$$

valid in $R$ to $\eta_{3}$ terms.

The process specified above will render, after $\gamma-\mathrm{I}$ factorizations, a full set of solutions of $L_{n}(y)=0$, say, $y_{i}(x)(i=\mathrm{I}, 2, \ldots n)$, analytic in $R(|x| \neq \infty)$ and such that

$$
\begin{gathered}
y_{i}(x) \sim e^{Q_{i}(x)} x^{r_{i}}{ }_{\gamma} \sigma_{i}(x) \\
\left({ }_{\gamma} \sigma_{i}(x) \text { a } \text { o-series } ; i=1,2, \ldots n ; x \text { in } R\right)
\end{gathered}
$$

to $\eta$ terms. For the present these functions are to be considered as possibly dependent on $\eta$. However, $\eta$ may be made as great as desired by suitably choosing $T(x)$ at the beginning of the construction of solutions.

Let $\tilde{y}_{i}(x) \quad(i=\mathbf{I}, 2, \ldots n)$ be another set of solutions for which the asymptotic relations $(2 \mathrm{I})$ are valid in $R$ to $\tilde{\eta}(>\eta)$ terms. We have

$$
\left(y_{i}^{(j-1)}(x)\right)=\left(e_{i, j}\right)\left(\widetilde{y}_{i}^{(j-1)}(x)\right) \quad(i, j=1, \ldots n)
$$

where $\left(c_{i, j}\right)$ is a matrix of constants. Now

$$
\left.\left(c_{i, j}\right)=\left(y_{i}^{(j-1)}(x)\right)\left(\check{y}_{i}^{(j-1)}(x)\right)^{-1}=y_{i}^{(j-1)}(x)\right)\left(\ddot{y}_{i, j}(x)\right)
$$

and

$$
c_{i, j}=\sum_{\lambda=1}^{n} y_{i}^{(i-1)}(x) \tilde{\tilde{y}}_{\lambda, j}(x) \quad(i, j=\mathrm{I}, 2, \ldots n) .
$$

In view of the asymptotic forms of the two sets of solutions it follows from $(22$ b) that

$$
\begin{gathered}
c_{i, j}=e^{Q_{i, j}(x)} x^{r_{i, j}}\left(\delta_{i, j}+\frac{\gamma_{x}^{i, j}(x)}{x^{\frac{x}{k}}}\right) \\
{\left[Q_{i, j}(x)=Q_{i}(x)-Q_{j}(x) ;\left(\delta_{i, j}\right)=I ; i, j=\mathrm{I}, 2, \ldots n\right]}
\end{gathered}
$$

where $\left|\gamma_{x}^{i, j}(x)\right| \leqq \gamma_{x}(x$ in $R), x$ is some positive number and $r_{i, i}=\mathrm{o}(i=\mathrm{I}, 2, \ldots n)$. Let $x$ recede to infinity within $R$. It will follow that

$$
c_{i, j}=\lim _{x} e^{Q_{i, j}(x)} x^{r_{i, j}}\left(\delta_{i, j}+\frac{\gamma_{x}^{i, j}(x)}{x^{\bar{k}}}\right),
$$


whenever the latter limit exists. In particular,

$$
c_{i, i}=\lim _{x}\left(\mathrm{I}+\frac{\gamma_{x}^{i, j}(x)}{x^{\frac{\alpha}{k}}}\right)=\mathrm{I} \quad(i=\mathrm{I}, 2, \ldots n) .
$$

By (2)

Hence, by (23a),

$$
\Re Q_{i, j}(x) \leqq 0 \quad(i<j)
$$

$$
c_{i, j}=0 \quad(i<j=\mathrm{I}, \ldots n)
$$

provided that $x$ is sufficiently great. This, however, can and will be supposed to be secured by. suitable choice of the set of solutions $\tilde{y}_{i}(x)(i=\mathrm{I}, 2, \ldots n){ }^{1}$ Accordingly,

$$
y_{i}(\dot{x})=\tilde{y}_{i}(x)+\sum_{\lambda=1}^{i-1} e_{i, \lambda} \tilde{y}_{\lambda}(x) \quad(i=\mathrm{I}, 2, \ldots n) .
$$

Consider the above relations for $i \leqq \Gamma_{1}$. Now, in virtue of $(2)$,

so that

$$
\begin{gathered}
\left|c_{i, \lambda}\right|=\lim _{x} e^{n Q_{i, \lambda}(x)}\left|\frac{\gamma_{\alpha}^{i, j}(x)}{\frac{x}{x^{k}-r_{i, \lambda}}}\right|=\lim _{x}\left|\frac{\gamma_{x}^{i, j}(x)}{\frac{x}{x^{\bar{k}}-r_{i, \lambda}}}\right|=0 \\
{\left[\lambda=\mathrm{I}, 2, \ldots i-\mathrm{I} ; i=\mathrm{I}, 2, \ldots \Gamma_{1}\right]}
\end{gathered}
$$

$$
y_{i}(x)=\tilde{y}_{i}(x)
$$

$\left(i=1,2, \ldots \Gamma_{1}\right)$.

But the functions (25) were obtained by iterations; that is, by the processes of Lemma 4. The results of that Lemma can therefore be completed as follows.

Let the asymptotic relations satisfied by the coefficients in $L_{n}(y)=0$ be in the ordinary sense. The Iteration process of Lemma 4 (\$6) will then yield results independent of the choice of $T(x)$. Accordingly, in this case, the asymptotic relations, resulting from the application of that Lemma, will be valid in the ordinary sense (that is, to infinity of terms).

With the above fact in view, we repeat the reasoning made from the beginning of this section. The asymptotic relations obtained in each consequtive step will be all in the ordinary sense, provided that integrations are suitably defined. This, however, is possible by virtue of the hypotheses made concerning $R$ and by virtue of Lemma 3. Hence there will exist a full set of solutions of $L_{n}(y)=0$ such that the relations (2 I) are satisfied in the ordinary sense.

Thus, the proof of the Theorem has been completed.

\footnotetext{
${ }^{1}$ Such a choice will give a suitably great value of $\bar{\eta}$.
} 
8. Extension of the Regions of Validity of the asymptotic Relations. Consider the Fundamental Existence Theorem of $\S 7$ as referring to a system (B) of $\S \mathrm{I}^{\circ}$, possessing a formal matrix solution $(7 ; \S \mathrm{I})$. In particular, the results of this section would apply to a single equation (A) $\left(\S I^{\circ}\right)$; in fact, we need only to note that with such an equation (A) there is associated a system (D) (§ I). Consider a pair of adjacent regions

$$
R_{\sigma}, R_{\sigma+1} \quad\left(\sigma=1,2, \ldots N ; G_{N+1}=R_{1}\right)
$$

of the set of regions $(16 \mathrm{a} ; \S 2)$. Let the regions of the latter set be ordered in the counter clockwise direction. The boundaries bounding $R_{\sigma}, R_{\sigma+1}$ will be in succession $B_{\sigma-1, \sigma}, B_{\sigma, \sigma+1}, B_{\sigma+1, \sigma+2}\left(B_{N, N+1}=B_{N, 1}=B_{1, N}\right.$; cf. $\S 2$ following (16 a)).

Corresponding to $R_{\sigma}$ there exists a matrix solution $Y^{\sigma}(x)=\left(y_{i, j}^{\sigma}(x)\right)$ such that the asymptotic relations (with respect to the power series in the $\sigma$-series factors of the formal solutions)

$$
Y^{\sigma}(x) \sim S(x)=\left(e^{Q_{i}(x)} x^{r_{i, j}} \sigma_{i, j}(x)\right)
$$

hold either in $R_{\sigma}$ or in a subregion $K_{\sigma}^{\prime \prime}$, of $R_{\sigma}$, possessing $B_{\sigma, \sigma+1}$ as one boundary and $B_{o-1, \sigma}^{\prime \prime}$ (with the limiting direction of $B_{\sigma-1, \sigma}$ ) as another boundary (cf. the Theorem of $\$ 7)$. Similarly, there exists a matrix solution $Y^{\sigma+1}(x)=\left(y_{i, j}^{\sigma+1}(x)\right)$ such that

$$
Y^{o+1}(x) \sim S(x)
$$

either in $R_{\sigma+1}$ or in $R_{\sigma+1}^{\prime \prime}$; the region $R_{\sigma+1}^{\prime \prime}$ (if there is an occasion to consider such a region) has $B_{\sigma, \sigma+1}$ for one boundary and it has $B_{\sigma+1, \sigma+2}^{\prime \prime}$ (with the limiting direction of $\left.B_{\sigma+1, \sigma+2}\right)$ for another boundary.

Let $R_{\sigma, \sigma+1}$ be a combined region (containing $B_{\sigma, \sigma+1}$ in its interior) over which the relations (2) and ( 2 a) are asserted in accordance with the Fundamental Existence Theorem. The right and left boundaries of $R_{\sigma, \sigma+1}$ can be taken as regular curves with the limiting direction of $B_{\sigma-1, \sigma}$ and $\boldsymbol{B}_{\sigma+1, \sigma+2}$, respectively. Suppose that in $R_{\sigma}$ there is an ordering $(2 ; \S 7)$.

The regions in which (2) and $\left(2\right.$ a) holds have the curve $B_{\sigma, \sigma+1}$ in common. Now

$$
Y^{\sigma}(x)=C^{\sigma} Y^{\sigma+1}(x) ; \quad C^{\sigma}=\left(c_{i, j}^{\sigma}\right) .
$$

The matrix of constants $C^{\sigma}$ will be investigated by means of the asymptotic relations (2), (2 a) which, for $x$ on $B_{\sigma, \sigma+1}$, yield the following:

$$
C^{\sigma}=Y^{\sigma}(x) Y^{\sigma+1^{-1}}(x) \sim\left(e^{Q_{i, j}(x)} x^{r^{\prime} i, j} \delta_{i, j}\right),
$$


where $Q_{i, j}(x)=Q_{i}(x)-Q_{j}(x), r_{i, j}^{\prime}=\mathrm{o}(i, j=1, \ldots n),\left(\delta_{i, j}\right)$ is the identity matrix and the asymptotic relations are with respect to the $\delta_{i, j}$. Letting $x \rightarrow \infty$ along $B_{\sigma, \sigma+1}$, we find the following.

The $c_{i, j}^{\sigma}=\mathrm{I}(i=\mathrm{I}, 2, \ldots n) . \quad A$ constant $c_{i, j}^{\sigma}(i \neq j)$ may be distinct from zero if and only if $\mathfrak{R} Q_{i, j}(x)$ approaches positive infinity along $B_{\sigma, \sigma+1}$. In particular, then, a constant $c_{i, j}^{\sigma}(i \neq j)$ will be zero when $i<j$, or when $i$ and $j$ are subscripts of the same logarithmic group, or when $\Re Q_{i, j}(x)$ is bounded on $B_{\sigma, \sigma+1}$.

Thus, from (3) it would follow that

$$
\left(y_{i, j}^{\sigma}(x)\right)=\left(y_{i, j}^{\sigma+1}(x)+\sum_{\lambda=1}^{i-1} c_{i, \lambda}^{\sigma} y_{\lambda, j}^{\sigma+1}(x)\right)
$$

On writing

$$
\left(y_{i, j}^{\sigma}(x)\right)=\left(e^{Q_{j}(x)} x^{r_{i, j}} \eta_{i, j}^{\sigma}(x)\right),\left(y_{i, j}^{\sigma+1}(x)\right)=\left(e^{Q_{i}(x)}, x^{r_{i, j}} \eta_{i, j}^{\sigma+1}(x)\right),
$$

where for $x$ within certain regions

$$
\left(\eta_{i, j}^{\sigma}(x)\right) \sim\left(\sigma_{i, j}(x)\right),\left(\eta_{i, j}^{\sigma+1}(x)\right) \sim\left(\sigma_{i, j}(x)\right)
$$

it is noted that

$$
\left(y_{i, j}^{\sigma}(x)\right)=\left(e^{Q_{i}(x)}\left[x^{r_{i, j}} \eta_{i, j}^{\sigma+1}(x)+\mathfrak{\Im}_{i, j}^{\sigma+1}(x)\right]\right)
$$

Here

$$
\left(\mathfrak{S}_{i, j}^{\sigma+1}(x)\right)=\left(\sum_{\lambda=1}^{i-1} c_{i, \lambda}^{\sigma} e^{Q_{\lambda, i}(x)} x^{r_{\lambda, j}} \eta_{\lambda, j}^{\sigma+1}(x)\right)
$$

In view of the italicized statement following (4) the $Q_{\lambda, i}(x)$ which actually enter in the second members of $(6$ a) have their real parts approaching negative infinity along $B_{\sigma, \sigma+1}$. Now $B_{\sigma, \sigma+1}$ is a regular curve; hence the above $\mathfrak{R} Q_{\lambda, i}(x)$, just referred to, approach negative infinity along $B_{\sigma, \sigma+1}$ essentially as $-c\left|x^{\gamma}\right|$ $(c>0, \gamma>0)$. None of the functions $\Re Q_{2, i}(x)$ vanish interior $R_{\sigma, \sigma+1}$. It is seen without difficulty that either the exponents, displayed in (6a), satisfy the relations

$$
\left|e^{Q_{\lambda, i}(x)}\right| \sim 0
$$

throughout the part of $R_{\sigma, \sigma+1}$ bounded by $B_{\sigma, \sigma+1}$ and the left boundary of $R_{\sigma, \sigma+1}$, or they satisfy these relations in the part of $R_{\sigma, \sigma+1}$ bounded by $B_{\sigma, \sigma+1}$ and bounded by a regular curve with the limiting direction of the left boundary of $R_{\sigma, \sigma+1}$. Denote this part of $R_{\sigma, \sigma+1}$ on and to the left of $B_{\sigma, \sigma+1}$ by $R_{\sigma, \sigma+1}^{l}$. 28-33617. Acta mathematica. 62. Imprimé le 8 novembre 1933. 
Since the $\left|\eta_{i, j}^{\sigma+1}(x)\right|$ cannot approach infinity faster than a power of $|\log x|$, it follows that

$$
\left(\Im_{i, j}^{\sigma+1}(x)\right) \sim(0)
$$$$
\left(x \text { in } R_{\sigma, \sigma+1}^{l}\right) \text {. }
$$

By virtue of (6), then, it becomes manifest that

$$
Y^{\sigma}(x) \sim S(x)
$$

not only in $K_{\sigma}$ (or $R_{u}^{\prime \prime}$, as the case may be ${ }^{1}$ ) but also in a subregion $R_{\sigma, \sigma+1}^{l}$ (as specified above) of $R_{\sigma+1}$, extending to the left of $R_{\sigma}$ and possessing a left boundary with the limiting direction of the left boundary of $R_{\sigma+1}$. The combined region of validity of $(8)$ will be denoted by $G_{\sigma}$ : $G_{\sigma}$ uill contain $B_{\sigma, \sigma+1}$ in the interior. The above result holds for $\sigma=\mathrm{I}, 2, \ldots \mathrm{V}$. Similarly, if in the above the words left and right are interchanged, it is seen that there exists a region ${ }_{1} G_{\sigma}$, containing $B_{\sigma, \sigma+1}$ in the interior, such that a matrix solution ${ }_{1} Y^{\sigma}(x)$ satisfies the asymptotic matrix relation

$$
{ }_{1} Y^{\sigma}(x) \sim S(x)
$$

for $x$ in ${ }_{1} G_{\sigma}(\sigma=1,2, \ldots N)$.

Now a region $G_{\sigma}$ will certainly possess in common with $G_{\sigma+1}$ a region $G_{\sigma, \sigma+1}$, bounded by regular curves; this being true for $a=\mathrm{I}, 2, \ldots N$. In the case when the curves $B_{\sigma, \sigma+1}, B_{\sigma+1, \sigma+2}$ have different limiting directions the truth of the above statement follows from the fact that the left boundary of $G_{\sigma}$ and the right boundary of $G_{\sigma+1}$ have the limiting directions of $B_{\sigma+1, \sigma+2}$ and $B_{\sigma, \sigma+1}$, respectively. In this case the boundaries of $G_{\sigma, \sigma+1}$ have different limiting directions. On the other hand, when the curves $B_{\sigma, \sigma+1}, B_{\sigma+1, \sigma+2}$ have the same limiting directions at infinity, the following is noted. The part of the region $G_{\sigma+1}$ which lies to the right of $B_{\sigma+1, \sigma+2}$ consists, in virtue of the Fundamental Existence Theorem, of the region bounded by $B_{\sigma, \sigma+1}$ and $B_{\sigma+1, \sigma+2}$, that is, this part consists of $R_{\sigma}$. In this case, then, $G_{\sigma, \sigma+1}$ will consist of the part of $R_{\sigma}$ bounded on the left by the left boundary of $G_{\sigma}$; the two regular curves bounding $G_{\sigma, \sigma+1}$ will certainly be distinct.

Consider two matrix solutions

$$
Y^{\sigma}(x), \quad Y^{\sigma+1}(x)
$$

\footnotetext{
${ }^{1}$ Here $R_{\sigma}^{\prime \prime}$, if used, has $B_{\sigma, \sigma+1}$ for its left boundary.
} 
of the type referred to in the italictzed statement made in connection with (8). ${ }^{1}$ We have $Y^{\sigma}(x)=C_{\sigma} Y^{\sigma+1}(x)\left[C_{\sigma}=\left(c_{\sigma: i, j}\right)\right]$. Some information concerning the constants $c_{\sigma: i, j}(i, j=\mathrm{I}, \ldots n)$ can be obtained ${ }^{2}$ by noting that, in view of the asymptotic relations satisfied by the matrices (9) in the regions $G_{\sigma}, G_{\sigma+1}$, respectively, we have

$$
C_{o}=\left(e_{o: i, j}\right)=Y^{\sigma}(x) Y^{\sigma+1^{-1}}(x) \sim\left(e^{Q_{i, j}(x)} x^{r^{\prime} i, j} \delta_{i, j}\right)
$$

for $x$ in $G_{\sigma, \sigma+1}$. Now $G_{\sigma, \sigma+1}$ is a subregion of $R_{\sigma+1}$; the ordering $(2 ; \xi 7)$ of the $\Re Q_{i}(x)$ is assumed ${ }^{3}$ in $R_{\sigma+1}$. By a reasoning of the type which has been applied to $C^{\sigma}$ it is presently established that $c_{\sigma: i, i}=\mathrm{I} \quad(i=\mathrm{I}, \ldots n)$. Moreover, a constant $c_{\sigma: i, j}(i \neq j)$ may be distinct from zero if and only if $\Re Q_{i, j}(x)$ approaches positive infinity along every regular cuvve in $G_{\sigma, \sigma+1}$. For, if $\mathfrak{R} Q_{i, j}(x)$ did not approach positive infinity along some regular curve $C$, in $G_{\sigma, \sigma+1}$, it would follow that $\left|e^{Q_{i, j}(x)}\right|$ is bounded on $C$; this, however, would imply, in view of (9a), that $c_{\sigma: i, j}=0$. In particular, $c_{\sigma: i, j}(i \neq j)=0$ when $i<j$ or when $i$ and $j$ are subscripts of the same logarithmic group.

9. Converse Problems. Consider a system (B) $\left(\S \mathrm{I}^{\circ}\right)$ and assume for de. finiteness that the ordering of the $\Re Q_{i}(x)(i=1,2, \ldots n)$, as specified by $(2 ; \$ 7)$, is maintained in the region $R_{1}(16 \mathrm{a} ; \S 2)$. Throughout this section it will be supposed that the $Q_{i}(x)$ in the consecutive rows of the formal matrix $S(x)$ occur in the same order as in $(2 ; \S 7)$. In view of $\$ 8$ and of the Fundamental Existence Theorem the following can be asserted.

Depending on the $Q_{i}(x) \quad(i=\mathrm{I}, \ldots n)$, only, the complete vicinity of infinity is divided into $N$ adjacent regions

$$
R_{1}^{\prime}, R_{2}^{\prime}, \ldots R_{N}^{\prime} . \quad\left(R_{N+1}^{\prime}=R_{1}^{\prime}\right)
$$

separated by regular curves

$$
B_{1,2}^{\prime}, B_{2,3}^{\prime}, \ldots B_{N-1, N}^{\prime} \quad\left(B_{N, N+1}^{\prime}=B_{N, 1}^{\prime}=B_{1, N}^{\prime}\right)
$$

A curve $B_{\sigma, \sigma+1}^{\prime}$ separates $R_{\sigma}^{\prime}$ from $R_{\sigma+1}^{\prime}$. This curve is sometimes coincident with $B_{\sigma, \sigma+1}$ (cf. $\$ 2$ ). In the case when $B_{\sigma, \sigma+1}^{\prime}$ is distinct from $B_{\sigma, \sigma+1}$ it extends

${ }_{1}$ Thus, $Y^{\sigma+1}(x)$ is to denote now a matrix possibly distinct from the matrix for which (2 a) had been asserted.

${ }^{2}$ Compare with (4) and the sequel.

${ }^{3}$ This is a matter of notation. 
to the left of $B_{\sigma, \sigma+1}$ (that is, it is in the counter clockwise direction ${ }^{1}$ from $\left.\boldsymbol{B}_{\sigma, \sigma+1}\right)$, it lies then interior $R_{\sigma+1}$; but in every case it has the limiting direction of $B_{\sigma, \sigma+1}$. Associated with the region $R_{\sigma}^{\prime}$ there exists a matrix solution $Y^{\sigma}(x)=\left(y_{i, j}^{\sigma}(x)\right)$ such that

$$
Y^{\sigma}(x) \sim S(x), \quad Y^{\sigma^{(1)}}(x) \sim S^{(1)}(x) \quad\left(x \text { in } R_{\sigma}^{\prime}\right) .
$$

Such a matrix can be constructed for $\sigma=1,2, \ldots N$.

If we start in $R_{1}^{\prime}$ with a certain determination of the elements of $S(x)$, and if $x$ describes a closed circuit containing the origin and extending across the regions $R_{\sigma}^{\prime} \quad(\sigma=\mathrm{I}, 2, \ldots N+\mathrm{I})$, we arrive to a new determination ${ }^{2}$ which will be associated with the region $R_{N+1}^{\prime}$. Here

$$
S^{\prime}(x)=L S(x), \quad L=\left(l_{i, j}\right),
$$

where the $l_{i, j}$ are certain constants ${ }^{3}$ essentially characterized by the $r_{i, 1}(i=\mathrm{r}, 2, \ldots n)$ and by the logarithmic groups. In view of these facts it is natural to define $Y^{N+1}(x)$, a matrix solution associated with $R_{N+1}^{\prime}$, as

$$
Y^{x+1}(x)=L Y^{\prime}(x)
$$

- The following definition will be now introduced.

Definition. Consider a singular system (B) of $\S \mathrm{I}^{\circ}$. The coefficients of the various powers of $x$ in the $Q_{i}(x)(i=1,2, \ldots n)$, involved in the corresponding formal matrix solution $S(x)$, as well as the constants of the matrix $L$, involved in (3), will be termed characteristic constants belonging to the singular point of the system.

The above definition and the consideration of characteristic constants, to follow, is suggested by Birkhoff's treatment of problems of the Riemann type, which he gave for the case when the roots of the characteristic equation are all distinct. ${ }^{4}$ Now, the characteristic constants specify not only the regions (I),

${ }^{1}$ In this section we continue to assume that the regions $(16 a ; \S 2)$ are ordered in the counter clockwise direction.

2 The values $Q_{i}(x)$ will be the same in the corresponding elements of $S(x)$ and $S^{\prime}(x)$.

${ }^{3}$ In the case when the roots of the characteristic equation are all unequal $L$ is of the form $\left(\delta_{i, j} \cdot \exp \left(2 \pi r_{i} \sqrt{-1}\right)\right.$.

4 G. D. Birkhoff, The Generalized Riemann Problem for Linear Differential Equations and the Allied Problems for Iinear Difference and $q$-Difference Equations, Proc. Am. Acad. Arts and Sciences, vol. 49 (1913) pp. 52I-568. This paper will be referred to as (B). In using or quoting the results of (B) the notation will be used conforming with that of the present paper. 
within which (2) holds, but they also essentially specify the nature of matrices of constants

$$
C_{\sigma}=\left(c_{\sigma: i, j}\right) \quad(\sigma=\mathrm{I}, \ldots N)
$$

involved in the relations

$$
Y^{\sigma}(x)=C_{\sigma} Y^{\sigma+1}(x) \quad(\sigma=\mathrm{I}, 2, \ldots N)
$$

here the matrices $Y^{\prime}(x), \ldots Y^{N+1}(x)$ are those occurring in (2) and (4). In fact, in view of relations (2) and (4) it follows that

$$
\begin{gathered}
C_{\sigma}=Y^{\sigma}(x) Y^{\sigma+1-1}(x) \sim\left(e^{Q_{i}(x)-Q_{j}(x)} x^{r^{\prime} i, j} \delta_{i j}\right) \\
{\left[x \text { on } B_{\sigma, \sigma+1}^{\prime} ; \sigma=\mathrm{I}, 2, \ldots N ; r_{i, i}^{\prime}=0 ; i=\mathrm{I}, 2, \ldots n\right] .}
\end{gathered}
$$

We note that the $Q_{i}(x)-Q_{j}(x)$ and the $r_{i, j}^{\prime}$ depend only on the characteristic constants.

In terms of the characteristic constants it is also possible to define a matrix

$$
T(x)=\left(x^{Q_{i}(x)} \eta_{i, j}(x)\right)
$$

possessing the following property. As the variable describes a closed circuit, containing the origin and extending in the counter clockwise direction from a point in $R_{\sigma}^{\prime}$ to $R_{N+1}^{\prime}$, the new determination $T^{\prime}(x)$ of $T(x)$ will be such that

$$
T^{\prime}(x)=L T(x)
$$

We do not need to know. $T(x)$ in detail. It will be noted that in the case treated in $(\mathbf{B})^{\mathbf{1}} T(x)=\left(\delta_{i, j} x^{r_{i}} x^{Q_{i}(x)}\right) ; T(x)$ of $(7)$ is an obvious generalization of the latter matrix. Furthermore, if the formal series $\gamma_{i, j}(x)$ are in negative integral powers of $x^{\frac{1}{k}}$ ( $k$ a suitable integer $)^{2}$ the formal matrix

$$
T(x)\left(\gamma_{i, j}(x)\right)=S(x)
$$

will be of the type of a formal matrix solution of a system of the type (B) $\left(\$ \mathrm{I}^{\circ}\right) .^{3}$

The following Theorem relating to a.simple converse problem will be proved.

1 (B; p. 548).

2 With the determinant of constant terms distinct from zero.

${ }^{3}$ That is, the elements of $S^{-1}(x) S^{(1)}(x)$ are formal series of the type $\left(\mathrm{I} ; \S \mathrm{I}^{\circ}\right)$. In the case treated in $(\mathbf{B})$ we would have $T(x)\left(\gamma_{i, j}(x)\right)=\left(\gamma_{i, j}(x) x^{r_{i}}, \exp Q_{i}(x)\right)$. 
Theorem I. Suppose that $S(x)$ is a formal matrix of the type which could possibly occur as a formal matrix solution associated with a system of type (B) $\left(\S \mathrm{I}^{\circ}\right)$. Let the regions (I) and the curves (I a) be specified by the polynomials $Q_{i}(x) \quad(\sigma=1,2, \ldots N)$ occurring in $S(x)$. Suppose there exist matrices $Y^{o}(x)$ $(\sigma=\mathrm{I}, 2, \ldots N)$ of analytic functions, satisfying the asymptotic relations (2). Assume moreover, that on writing $(4)$ and $\left(5\right.$ a) the elements in the matrices $C_{\sigma}(\sigma=1,2, \ldots N)$ are constants. It follows then that there exists a singular system (B) which is satisfied by the matrix solutions $Y^{\sigma}(x) \quad(\sigma=1,2, \ldots N)$.

In fact, on letting

$$
Y^{\sigma}(1)(x)=Y^{\sigma}(x) A^{\sigma}(x) \quad(\sigma=1,2, \ldots N)
$$

it follows, in view of the assymptotic relations (2), that

$$
\begin{gathered}
A^{\sigma}(x)=Y^{\sigma-1}(x) Y^{\sigma(1)}(x) \sim\left(\alpha_{i, j}^{\sigma}(x)\right) \\
\left(x \text { in } R_{\sigma}^{\prime} ; \quad \sigma=1,2, \ldots N\right) ;
\end{gathered}
$$

here the $\alpha_{i j}^{\sigma}(x)$ are formal series of the type $\left(\mathrm{I} ; \S \mathrm{I}^{\circ}\right)$. Now by virtue of $(5 \mathrm{a})$

$$
A^{\sigma}(x)=Y^{\sigma+1^{-1}}(x) C_{\sigma}^{-1} C_{\sigma} Y^{\sigma+1^{(1)}}(x)=Y^{\sigma+1^{-1}}(x) Y^{\sigma+1^{(1)}}(x)=A^{\sigma+1}(x) .
$$

Accordingly, $A^{\sigma}(x)$ is independent of $\sigma$ and we may write $A^{\sigma}(x)=A(x)$. The matrix equation $Y^{(1)}(x)=Y(x) A(x)$ is of type (B) $\left(\S \mathrm{r}^{\circ}\right)$ and constitutes the required system.

A deeper lying result, which is of the Riemann type, is embodied in the following Theorem. ${ }^{1}$

Theorem II. Let a set of characteristic constants be preassigned ( $f$. Definition). These constants define regions ( $\mathrm{I}$ ) and curves (I a). Let matrices of constants $C_{o}$ be associated with the curves $B_{\sigma, \sigma+1}^{\prime}(\sigma=\mathrm{I}, 2, \ldots N)$, respectively. A matrix $C_{o}$ will be assumed to satisfy a condition, depending on the characteristic constants,

$$
\begin{gathered}
C_{\sigma} \sim\left(e^{Q_{i}(x)-Q_{j}(x)} x^{r^{\prime} i, j} \delta_{i, j}\right) \\
{\left[x \text { on } B_{\sigma, \sigma+1}^{\prime} ; \quad \sigma=\mathrm{I}, 2, \ldots N ; \quad r_{i, i}^{\prime}=0 ; \quad i=\mathrm{I}, \ldots n\right] .}
\end{gathered}
$$

It follows then that there exist matrices of functions $Y^{(\sigma)}(x)$ such that $(5$ a) holds, while

${ }_{1}^{1}$ This theorem constitutes an extension to the unrestricted case of a theorem in (B; pp. $548-550)$.

${ }_{2}$ That is, we have a set of constants of the type of a set of characteristic constants of a system (B) 


$$
\begin{gathered}
Y^{\sigma}(x) \sim S(x)=\left(e^{Q_{i}(x)} x^{r_{i}} \sigma_{i, j}(x)\right) \\
\left(x \text { in } R_{\sigma}^{\prime} ; \sigma=\mathrm{I}, 2, \ldots N+\mathrm{I}\right),
\end{gathered}
$$

where $S(x)$ is of the type of a formal matrix solution of a system of type $(\mathrm{B})\left(\S \mathrm{I}^{\circ}\right)$.

Before proceeding with the proof it will be of interest to note that, in view of Theorem I, this theorem implies that a given set of characteristic constants determines a singular differential system (B).

Use will be made of the following theorem proved in $(B)^{1}$, which will be stated with some slight change in notation.

'Let $K_{1}, \ldots K_{N}$ be $N$ simple closed analytic curves in the extended complex plane. Let $A_{1}(x), \ldots A_{N}(x)$ be matrices of functions defined and indefinitely differentiable along $K_{1}, \ldots K_{N}$ of determinant not zero. If furthermore at any point of intersection of $K_{\alpha}, K_{\beta}$ the matrices $A_{a}(x), A_{\beta}(x)$ are such that formal derivatives of all orders of the matrix

$$
A_{\alpha}(x) A_{\beta}(x)-A_{\beta}(x) A_{\alpha}(x)
$$

vanish, there exists a matrix $\Phi(x)$ with the following properties:

(I) each element of $\Phi(x)$ is analytic except along $K_{1}, \ldots K_{N}$ and at an arbitrary point $x=\alpha_{0}$ where the elements may become infinite to finite order; $|\Phi|$ nowhere vanishes save possibly at $x=\alpha_{0}$;

(2) the elements of $\Phi(x)$ are continuous and indefinitely differentiable along each curve $K_{i}$ from either side, analytic from either side save at the points of intersection of these curres, or at those points where an element of $(x-\alpha)^{l} A_{i}(x)$ [or $x^{-l} A_{i}(x)$ if $\alpha_{0}=\infty$ ] is indefinitely differentiable for a suitable $l$.

(3) if a + and - side of each curve $K_{\sigma}$ is chosen, then

$$
\Phi\left(x_{\sigma}^{+}\right)=A_{\sigma}\left(x_{\sigma}\right) \Phi\left(x_{\sigma}^{-}\right) \quad(\sigma=\mathrm{I}, \ldots N),
$$

where $x_{\sigma}$ is on $K_{\sigma}, x_{\sigma}^{+}$is $x_{\sigma}$ when considered on the + side of $K_{\sigma}$, and $x_{\sigma}^{-}$is $x_{\sigma}$ when considered on the left side $K_{\sigma}$.'

Let $\varrho$ be a suitably great positive number. $K_{\sigma}$ will be defined as a simple curve with a continuously turning tangent, extending to infinity in two directions, and, outside of the circle $|x|=\varrho$, consisting of $B_{\sigma, \sigma+1}^{\prime}$ and of another regular curve $\bar{B}_{\sigma, \sigma+1}^{\prime}$. Such a construction can be effected for $\sigma=\mathrm{I}, 2, \ldots N$ in such a manner that the curves $K_{1}, \ldots K_{N}$ intersect only at $x=0$ and at $x=\infty$;

${ }^{1}$ (B; pp. 533-534). 
moreover, they can be so specified ${ }^{1}$ that the above theorem is applicable even though the curves, just constructed, have been subjected to conditions slightly weaker than those of the quoted theorem; however, the results of that theorem will continue to apply for $|x|>\rho$, at least.

Matrices $A_{\sigma}(x)$ will be defined as follows:

$$
\begin{array}{cc}
A_{\sigma}(x)=T^{-1}(x) C_{\delta} T(x) & \left(x \text { on } B_{\sigma, \sigma+1}^{\prime}\right), \\
A_{\sigma}(x)=I & \left(x \text { on } \bar{B}_{\sigma, \sigma+1}^{\prime}\right) .
\end{array}
$$

Here $T(x)$ depends on the characteristic constants only and is given by $(7)$. In the part of $K_{\sigma}$ interior the circle $|x|=\rho A_{\sigma}(x)$ is defined to satisfy the conditions of the quoted theorem (for $\sigma=\mathrm{I}, 2, \ldots N$ ). ${ }^{2}$ The point $x=\alpha_{0}$ may be taken, for instance, at the origin. At infinity $A_{\sigma}(x)$ will satisfy the conditions of the theorem. In fact, by (7) and (9), on writing

and noting that

$$
T^{-1}(x)=\left(e^{-\zeta_{j}(x)} \eta_{i, j}(x)\right)
$$

$$
\begin{aligned}
& C_{\sigma}=\left(e^{Q_{i}(x)-Q_{j}(x)} x^{r^{\prime} i, j}\left(\delta_{i, j}+o_{i, j}(x)\right)\right), \\
& \left(o_{i, j}(x)\right) \sim(0) \quad\left(x \text { on } B_{\sigma, \sigma+1}^{\prime}\right),
\end{aligned}
$$

we have

$$
A_{\sigma}(x)=B_{\sigma}(x)+C_{\sigma}(x)
$$

$$
\begin{aligned}
B_{\sigma}(x)= & \left(\sum_{\lambda_{1}, \lambda_{2}} \bar{\eta}_{i, \lambda_{1}}(x) x^{r^{\prime} \lambda_{1}, \lambda_{2}} \delta_{\lambda_{1}, \lambda_{2}} \eta_{\lambda_{2}, j}(x)\right)=\left(\sum_{2=1}^{n} \bar{\eta}_{i, \lambda}(x) \eta_{i, j}(x)\right)=I \\
& O_{\sigma}(x)=\left(\sum_{\lambda_{1}, \lambda_{2}} \bar{\eta}_{i, \lambda_{1}}(x) x^{r^{\prime} \lambda_{1}, \lambda_{2}} o_{\lambda_{1}, \lambda_{2}}(x) \eta_{\lambda_{2}, j}(x)\right) \sim(0) \quad\left(x \text { on } B_{\sigma, \sigma+1}^{\prime}\right) .
\end{aligned}
$$

Thus, along $B_{\sigma, \sigma+1}^{\prime}$,

$$
A_{\sigma}(x) \sim I
$$

There is no difficulty in showing that of $\bar{B}_{\sigma, \sigma+1}^{\prime}$.

${ }^{1}$ If $b_{\sigma}$ is the limiting direction of $B_{\sigma, \sigma+1}^{\prime}$ we shall take $b_{\sigma}+x$ for the limiting direction

$$
{ }^{2} \text { For the purposes at hand analyticity of the elements of } A_{\sigma}(x) \text { along the part of } K_{\sigma} \text { in- }
$$
terior the circle $|x|=\rho$ (points of the circle included) is not necessary. At the points of the circle (and interior, as well) indefinite differentiability is sufficient. 
(14a).

$$
\lim _{|x|} A_{\sigma}^{(m)}(x)=(0) \quad(m=\mathrm{r}, 2, \ldots)^{1}
$$

when $|x|$ approaches infinity along $B_{\sigma, \sigma+1}^{\prime}$.

There will exist a matrix $\Phi(x)=\left(\varphi_{i, j}(x)\right)$ with elements analytic for $|x|>\boldsymbol{g}$ except along $\boldsymbol{B}_{\sigma, \sigma+1}^{\prime}(\sigma=1,2, \ldots N)$ and except at $x=\infty$; moreover, $|\boldsymbol{\Phi}(x)| \neq 0$ $(|x|>\boldsymbol{\rho})$. Along $B_{\sigma, \sigma+1}^{\prime}$ the $\varphi_{i, j}(x)$ will be analytic from either side $(|x|>\boldsymbol{Q}$; $x \neq \infty$ ). Furthermore, on letting the right side of $\boldsymbol{B}_{\sigma, \sigma+1}^{\prime}$ (as viewed from the origin) be the + side, by virtue of (II) and (I 2 ) it will follow that

$$
\begin{gathered}
\Phi\left(x_{\sigma}^{+}\right)=T^{-1}\left(x_{\sigma}\right) C_{\sigma} T\left(x_{\sigma}\right) \boldsymbol{\Phi}\left({ }_{\sigma}\right) \\
\left(x_{\sigma} \text { on } B_{\sigma, \sigma+1}^{\prime} ; \sigma=\mathrm{I}, 2, \ldots N\right) .
\end{gathered}
$$

We have of course $T\left(x_{\sigma}\right)=T\left(x_{\sigma}^{+}\right)=T\left(x_{\sigma}^{-}\right)$.

Let

$$
Y^{\sigma}(x)=T(x) \Phi(x) \quad\left(x \text { in } R_{\pi}^{\prime} ; \sigma=\mathrm{I}, 2, \ldots N+\mathrm{I}\right) .
$$

In view of (I5) the matrices $Y^{\circ}(x)$, as defined in (I6), will be connected by the relations ( 5 a). Now, from the treatment of the Riemann problems in (B) it follows that

$$
\left(\varphi_{i, j}(x)\right) \sim\left(\gamma_{i, j}(x)\right) \quad\left(x \text { in } R_{\sigma}^{\prime} ; \sigma=\mathrm{I}, \ldots N\right)
$$

where the formal series $\gamma_{i, j}(x)$ are of the type of those in $(7 \mathrm{~b})$; as a consequence of (14) and (I5) these formal series are independent of $\sigma$. On taking account of the statement made in connection with $(7 \mathrm{~b})$, it follows by virtue of ( 16$)$ and (I7) that the assymptotic relations ( 10 ) are satisfied. This completes the proof of our theorem.

On the basis of 'Theorem II it is possible to solve the 'Generalized Riemann Problem':

To construct a differential system with prescribed singular points $x_{1}, x_{2}, \ldots x_{q}$; the system to be of singular type $\left(\mathrm{cf} . \S \mathrm{I}^{\circ}\right)$ in the neighborhood of each of the above points. Moreover, the system is to possess a given monodromic group, the characteristic constants being preseribed at each singular point.

A problem of this type had been formulated and solved in $(B)^{2}$ under the assumption that the characteristic constants are of the type which may occur in a differential system, the roots of whose characteristic equation are all distinct

1 The superseript in (14a) denotes the $m$-th derivative.

${ }^{2}$ (B; pp. $55 \mathrm{I}-553$ ).

29-33617. Acta mathematica. 62. Imprimé le 20 december 1933. 
(this being true for each singular point). In the present formulation no such restriction is implied.

On the basis of the preceding the problem can be solved, without any additional difficulty, following the lines of the corresponding proof in $(B)$. The possibility of this problem rests intrinsically on the Fundamental Existence Theorem of $\$ 7$. 